\title{
Global existence for an age and spatially structured haptotaxis model with nonlinear age-boundary conditions
}

\author{
CHRISTOPH WALKER \\ Leibniz Universität Hannover, Institut für Angewandte Mathematik, Welfengarten 1, D-30167 Hannover, Germany \\ email: walker@ifam.uni-hannover.de
}

(Received 16 October 2007; revised 31 January 2008)

\begin{abstract}
A model focusing on key components involved in tumour invasion is studied. Tumour cell migration is based on cell motility and haptotaxis, i.e., the directed migratory response of tumour cells up gradients of cell-adhesion molecules. Individual cell processes are modelled according to cell age and several tumour phenotypes are incorporated. Global existence and uniqueness of nonnegative solutions to the corresponding coupled system of nonlinear partial differential equations are shown.
\end{abstract}

\section{Introduction}

The mathematical model considered in the present paper describes the early vascularised stage of tumour growth when the tumour begins to invade the surrounding healthy tissue. The basic biological assumptions are the following: The tumour is contained in a region of tissue and a blood supply has just been established. Tumour cells produce an enzyme that diffuses in the tissue region and degrades the extracellular matrix (ECM) locally. As well as making space into which tumour cells can move by simple diffusion, this produces oxygen (and other nutrients) essential for tumour survival and growth. The degradation of the ECM also results in a gradient of cell-adhesion molecules. Therefore, while the ECM may constitute a barrier to normal cell movement, it also provides a substrate to which tumour cells may adhere and upon which they may move. This directed migration of tumour cells up gradients of bound cell-adhesion molecules is called haptotaxis [10, 11].

The subsequent model is derived from the hybrid discrete-continuous model proposed in [5], but is continuous in all variables, and the individual processes of cells are modelled according to cell age. The model focuses on five key components involved in tumour invasion: the population densities for proliferating and quiescent tumour cells, the density of surrounding tissue macromolecules, the concentration of matrix degradative enzyme, and the concentration of oxygen. Oxygen could be representative of any nutrient or nutrients in general necessary for tumour cell survival. Proliferating and quiescent tumour cells are distinguished by position $x \in \Omega$, where $\Omega$ denotes the region of tissue, and by age $a \in(0, \infty)$. Age for proliferating tumour cells corresponds to the position in the cell cycle and if a cell divides, then both daughter cells have age zero. Age for quiescent cells corresponds to a rested position in the cell cycle (the age of a quiescent cell is fixed 
at the age it had when it changed from proliferation to quiescence, and if a quiescent cell changes back to proliferating, then aging resumes). Moreover, to account for tumour heterogeneity proliferating and quiescent tumour cells are also distinguished by type $j=1, \ldots, N$ corresponding to mutations with different characteristics and thus different aggressiveness. For instance, cell phenotypes may vary with respect to division and mutation rates, death rates, transition rates from proliferation to quiescence, recruitment rates from quiescence to proliferation, ECM-degrading enzyme productions, haptotactic migration rates, and oxygen consumption. We allow for a random mutation scheme, that is, a cell of type $j \in\{1, \ldots, N\}$ may divide into any other type $l \in\{1, \ldots, N\}$.

The dependent variables of the model are:

$p_{j}(t, a, x)$ : density of proliferating tumour cells of type $j$ at time $t$, position $x$, and age $a$,

$q_{j}(t, a, x)$ : density of quiescent tumour cells of type $j$ at time $t$, position $x$, and age $a$,

$f(t, x)$ : density of surrounding tissue macromolecules at time $t$ and position $x$,

$m(t, x)$ : matrix degradative enzyme concentration at time $t$ and position $x$,

$w(t, x) \quad$ : oxygen concentration at time $t$ and position $x$.

Clearly, all the variables are nonnegative. The vectors

$$
\begin{aligned}
& P(t, x):=\left(\int_{0}^{\infty} p_{1}(t, a, x) \mathrm{d} a, \ldots, \int_{0}^{\infty} p_{N}(t, a, x) \mathrm{d} a\right)^{T}, \\
& Q(t, x):=\left(\int_{0}^{\infty} q_{1}(t, a, x) \mathrm{d} a, \ldots, \int_{0}^{\infty} q_{N}(t, a, x) \mathrm{d} a\right)^{T}
\end{aligned}
$$

represent, respectively, the total population densities of proliferating and quiescent tumour cells for each phenotype. In order to describe the aforementioned processes the following equations were introduced in [32] which in turn are based on the model proposed in [5]: The matrix degradative enzyme breaks down the ECM upon contact and hence

$$
\partial_{t} f=-\underbrace{k(x) m f}_{\text {degradation }}, \quad t>0, \quad x \in \Omega,
$$

for some function $k \geqslant 0$. The enzyme produced by the tumour cells diffuses throughout the tissue and undergoes some form of decay:

$$
\partial_{t} m=\underbrace{\nabla_{x} \cdot\left(\alpha(x) \nabla_{x} m\right)}_{\text {diffusion }}+\underbrace{\Theta(P, Q)}_{\text {production }}-\underbrace{h(x) m}_{\text {decay }}, \quad t>0, \quad x \in \Omega,
$$

where $\alpha>0, \Theta \geqslant 0, h \geqslant 0$. Oxygen is assumed to be produced by the decay of the ECM, diffuses in space, is consumed by the tumour cells, and decays naturally:

$$
\partial_{t} w=\underbrace{\nabla_{x} \cdot\left(\beta(x) \nabla_{x} w\right)}_{\text {diffusion }}+\underbrace{\Gamma(f)}_{\text {production }}-\underbrace{\Lambda(P, Q) w}_{\text {uptake }}-\underbrace{e(x) w}_{\text {decay }}, \quad t>0, \quad x \in \Omega,
$$

where $\beta>0, \Gamma, \Lambda, e \geqslant 0$. Quiescent tumour cells do not age, but diffuse in space, enter from or transition back to proliferation, and are subject to cell death. These processes 
may be affected by the total population densities of tumour cells and by the ECM or oxygen concentration, respectively:

$$
\partial_{t} q_{j}=\underbrace{\nabla_{x} \cdot\left(\gamma_{j}(f) \nabla_{x} q_{j}\right)}_{\text {cell motility }}+\underbrace{\sum_{j}(a, w, P, Q) p_{j}}_{\text {enter from proliferation }}-\underbrace{\Phi_{j}(a, w, P, Q) q_{j}}_{\text {exit to proliferation }}-\underbrace{\Upsilon_{j}(a, w, P, Q) q_{j}}_{\text {cell death }}
$$

for $j=1, \ldots, N, t>0, a>0$, and $x \in \Omega$, where $\gamma_{j}>0, \Sigma_{j}, \Phi_{j}, \Upsilon_{j} \geqslant 0$. Proliferating tumour cells are subject to cell aging, cell division, cell death, and may enter from or exit to quiescence. Tumour cell migration is supposed to be due to random motility and haptotaxis in response to ECM gradients. These processes again may be affected by the total population densities of tumour cells and the ECM or oxygen concentration, respectively:

$$
\begin{aligned}
\partial_{t} p_{j}= & \underbrace{-\partial_{a} p_{j}}_{\text {cell aging }}+\underbrace{\nabla_{x} \cdot\left(\delta_{j}(f) \nabla_{x} p_{j}\right)}_{\text {cell motility }}-\underbrace{\nabla_{x} \cdot\left(p_{j} \chi_{j}(f) \nabla_{x} f\right)}_{\text {haptotaxis }}+\underbrace{\Phi_{j}(a, w, P, Q) q_{j}}_{\text {enter from quiescence }} \\
& -\underbrace{\sum_{j}(a, w, P, Q) p_{j}}_{\text {exit to quiescence }}-\underbrace{\Psi_{j}(a, w, P, Q) p_{j}}_{\text {cell death }}-\underbrace{b_{j}(a, w, P, Q) p_{j}}_{\text {cell division }},
\end{aligned}
$$

for $j=1, \ldots, N, t>0, a>0$, and $x \in \Omega$, where $\delta_{j}>0, \Psi_{j}, \chi_{j}, b_{j} \geqslant 0$. The terms on the right side of (1.2)-(1.5) depend locally on the dependent variables. That is, $\Theta(P, Q)$ in (1.2), for instance, has to be read as $\Theta(P(t, x), Q(t, x))$ etc., and we drop the arguments $t$ and $x$ merely for notational simplicity. Equation (1.5) is supplemented with age-boundary conditions taking into account that cells with phenotype $j$ and age 0 can be created by division of any cell type $l \in\{1, \ldots, N\}$ of any age $a>0$ :

$$
p_{j}(t, 0, x)=2 \sum_{l=1}^{N} \int_{0}^{\infty} b_{l}(a, w, P, Q) \psi_{l, j}(a, w, P, Q) p_{l}(t, a, x) \mathrm{d} a,
$$

for $j=1, \ldots, N, t>0$, and $x \in \Omega$, where $b_{l} \geqslant 0$ denotes the division rate of phenotype $l$, and $\psi_{l, j} \geqslant 0$ is the fraction of daughter cells of dividing mother cells of type $l$ with type $j$ mutation. This distribution satisfies

$$
\sum_{j=1}^{N} \psi_{l, j}=1, \quad l=1, \ldots, N
$$

Matrix degradative enzyme, oxygen, and tumour cells are assumed to remain within the tissue region and thus no-flux boundary conditions are assumed on the boundary $\partial \Omega$. Denoting by $v$ the outer unit normal on $\partial \Omega$ we assume that

$$
\partial_{v} m=\partial_{v} w=\partial_{v} q_{j}=\delta_{j}(f) \partial_{v} p_{j}-p_{j} \chi_{j}(f) \partial_{v} f=0, \quad j=1, \ldots, N
$$

The equations are supplemented with initial conditions $(j=1, \ldots, N)$

$$
f(0, \cdot)=f^{0}, \quad m(0, \cdot)=m^{0}, \quad w(0, \cdot)=w^{0}, \quad q_{j}(0, \cdot, \cdot)=q_{j}^{0}, \quad p_{j}(0, \cdot, \cdot)=p_{j}^{0} .
$$


To date, equations (1.1)-(1.8) have not been considered in this full generality. First versions of these equations were introduced in [5] and numerical results were presented therein. The derivation of the model and numerical results were also presented in [25] and subsequently in [7]. We refer to these papers for a more thorough biological background and an extensive list of related research. In [14] a similar model with linear age-boundary conditions was studied in which equation (1.1) involves a bounded non-local term and also a smoothing effect due to a diffusion term. The same problem with additional size structure and several phenotypes was investigated in [15]. The equations (1.1)-(1.8) were mathematically analyzed in [30] when age structure was neglected, only one phenotype was considered, and diffusion was linear. For this simplified case, global existence and uniqueness of nonnegative classical solutions were shown and numerical results were presented emphasizing the importance of haptotaxis in the cell migration process. These results were extended in [29] to include age structure. Therein one phenotype and linear diffusion were considered and the resulting age-boundary condition was taken to be linear, that is, the birth rate was depending merely on age but not on oxygen or the tumour cell densities. The main difficulty in this context arises from the interaction of the hyperbolic aging and the parabolic diffusion term together with the nonlinear haptotaxis term in the corresponding equation (1.5). In [29] the global well-posedness was shown for this case.

Clearly, age structured population models with diffusion have been considered for many years and different approaches have been used to analyze them mathematically. For example we refer to $[12,13,20,21,22,26,27,31]$. The novelty-and, at the same time, the main difficulties - of equations (1.1)-(1.8) is the combination of age and spatial structure together with the nonlinear haptotaxis term. Also, unlike in many of the other research papers, we include nonlinear diffusion and nonlinear age-boundary conditions. It is the aim of the present paper to prove the global well-posedness of equations (1.1)-(1.8). Of course, the model above is just one model among many that aims at describing tumour invasion, e.g., see $[6,9,23]$ and the references therein. However, the model considered herein captures all the features it is supposed to as shown by the numerical results recently presented in the journal Cell [7]. An additional motivation is to show how to deal mathematically with age and spatially structured population models with nonlinear diffusion and nonlinear age-boundary conditions that also include taxis terms. Thus, the present paper might provide a template for handling a broader range of similar models.

To simplify notation we write

$$
p:=\left(p_{1}, \ldots, p_{N}\right)^{T}, \quad q:=\left(q_{1}, \ldots, q_{N}\right)^{T}
$$

and define

$$
\begin{aligned}
\nabla_{x} \cdot\left(\gamma(f) \nabla_{x} q\right) & :=\left(\nabla_{x} \cdot\left(\gamma_{1}(f) \nabla_{x} q_{1}\right), \ldots, \nabla_{x} \cdot\left(\gamma_{N}(f) \nabla_{x} q_{N}\right)\right)^{T}, \\
\nabla_{x} \cdot\left(\delta(f) \nabla_{x} p\right) & :=\left(\nabla_{x} \cdot\left(\delta_{1}(f) \nabla_{x} p_{1}\right), \ldots, \nabla_{x} \cdot\left(\delta_{N}(f) \nabla_{x} p_{N}\right)\right)^{T}, \\
\nabla_{x} \cdot\left(p \chi(f) \nabla_{x} f\right) & :=\left(\nabla_{x} \cdot\left(p_{1} \chi_{1}(f) \nabla_{x} f\right), \ldots, \nabla_{x} \cdot\left(p_{N} \chi_{N}(f) \nabla_{x} f\right)\right)^{T} .
\end{aligned}
$$

Moreover, we introduce the matrix notation

$$
\Xi(a, w, P, Q):=\operatorname{diag}\left[\Xi_{1}(a, w, P, Q), \ldots, \Xi_{N}(a, w, P, Q)\right], \quad \Xi \in\{\Sigma, \Phi, \Psi, \Upsilon, b\}
$$


and

$$
\mathfrak{b}(a, w, P, Q):=\left[b_{l}(a, w, P, Q) \psi_{l, j}(a, w, P, Q)\right]_{1 \leqslant j, l \leqslant N}
$$

Then we can write (1.1)-(1.8) in more compact vector form to obtain the following system:

$$
\begin{aligned}
\partial_{t} f= & -k(x) m f \\
\partial_{t} m= & \nabla_{x} \cdot\left(\alpha(x) \nabla_{x} m\right)+\Theta(P, Q)-h(x) m \\
\partial_{t} w= & \nabla_{x} \cdot\left(\beta(x) \nabla_{x} w\right)+\Gamma(f)-\Lambda(P, Q) w-e(x) w \\
\partial_{t} q= & \nabla_{x} \cdot\left(\gamma(f) \nabla_{x} q\right)+\Sigma(a, w, P, Q) p-\Phi(a, w, P, Q) q-\Upsilon(a, w, P, Q) q \\
\partial_{t} p= & -\partial_{a} p+\nabla_{x} \cdot\left(\delta(f) \nabla_{x} p\right)-\nabla_{x} \cdot\left(p \chi(f) \nabla_{x} f\right) \\
& +\Phi(a, w, P, Q) q-\Sigma(a, w, P, Q) p-\Psi(a, w, P, Q) p-b(a, w, P, Q) p
\end{aligned}
$$

for $t>0, x \in \Omega$, and $a>0$, subject to the age-boundary conditions

$$
p(t, 0, x)=2 \int_{0}^{\infty} \mathfrak{b}(a, w, P, Q) p(t, a, x) \mathrm{d} a, \quad t>0, \quad x \in \Omega,
$$

no-flux conditions on $\partial \Omega$

$$
\partial_{v} m=\partial_{v} w=0, \quad \partial_{v} q=\delta(f) \partial_{v} p-p \chi(f) \partial_{v} f=0, \quad t>0,
$$

(with now obvious interpretation of $p \chi(f))$ and initial conditions

$$
f(0, \cdot)=f^{0}, \quad m(0, \cdot)=m^{0}, \quad w(0, \cdot)=w^{0}, \quad q(0, \cdot, \cdot)=q^{0}, \quad p(0, \cdot \cdot \cdot)=p^{0} .
$$

As mentioned earlier the main mathematical challenge is due to equation (1.18), which involves parabolic and hyperbolic terms, and the nonlinear age-boundary condition (1.19). To give a flavour of the main issues and to outline the present paper we fix suitable functions $f=f(t, x), w=w(t, x), P=P(t, x), Q=Q(t, x)$ and define temporarily

$$
\delta(t, x):=\delta(f(t, x)), \quad \mathfrak{b}(t, a, x):=\mathfrak{b}(a, w(t, x), P(t, x), Q(t, x)) .
$$

Then, a necessary first step is to understand the homogeneous problem

$$
\dot{p}+\mathbb{A}(t) p=0, \quad t>0,
$$

that is associated with (1.18). Here the operator $\mathbb{A}(t)$ is formally given by

$$
\mathbb{A}(t) p:=\partial_{a} p-\nabla_{x} \cdot\left(\delta(t, \cdot) \nabla_{x} p\right)
$$

where $p=p(a, x)$ is subject to no-flux conditions on $\partial \Omega$, i.e., $\partial_{v} p(a, x)=0$ for $a>0$, $x \in \partial \Omega$, and age-boundary conditions

$$
p(0, x)=2 \int_{0}^{\infty} \mathfrak{b}(t, a, x) p(a, x) \mathrm{d} a, \quad x \in \Omega .
$$

As shown in [29] the operator $-\mathbb{A}(t)$ generates for fixed $t$ a strongly continuous positive 
semigroup on $L_{1}\left((0, \infty), L_{\varrho}\left(\Omega, \mathbb{R}^{N}\right)\right), 1<\varrho<\infty$, possessing suitable smoothing properties with respect to the space variable (see Subsection A.2), which allow for a handling of the haptotaxis term. However, its time-dependent domain $D(\mathbb{A}(t))$ is not explicitly known. Thus, the standard theory (e.g., [16, 18, 19, 24]) for hyperbolic operators does not seem to apply in order to obtain a strongly continuous evolution system. Nevertheless, in the next section (see in particular Subsection 2.1) we will introduce an evolution system for $(1.22)-(1.23)$ on $L_{1}\left((0, \infty), L_{\varrho}\left(\Omega, \mathbb{R}^{N}\right)\right), 1<\varrho<\infty$. Since there is no general theory we can refer to, we will have to prove the relevant properties explicitly. The proofs can be found in the Appendix. The existence of the evolution operator then allows us to introduce a meaningful notion of a mild solution for equation (1.18) and to relate it to the operator $\mathbb{A}(t)$ studied in [29]. Of course, of particular interest in order to prove the existence of solutions to (1.14)-(1.21) is a precise understanding of how the evolution system depends on the previously fixed functions $f, w, P$, and $Q$. Having collected all the necessary tools in Section 1.2 we will demonstrate in Section 1.3 how to apply these in order to obtain the global well-posedness of (1.14)-(1.21). As mentioned above, some of the technical proofs related to the evolution operator will be postponed to the Appendix.

We conclude the introduction with a summary of our main result which can be paraphrased as follows:

Theorem. Let $\Omega \subset \mathbb{R}^{n}, n \leqslant 3$, be a bounded and smooth domain, and let the data $k, h$, $e, \chi, \Theta, \Lambda, \Gamma, \Sigma, \Phi, \Psi, \Upsilon, b, \psi$, be smooth, bounded, and nonnegative. Suppose that $\alpha, \beta$, $\gamma$, and $\delta$ are smooth, bounded, and positive. Given $\varrho>n$ and any nonnegative initial value $\left(f^{0}, m^{0}, w^{0}, q^{0}, p^{0}\right)$ in the space

$$
X:=W_{\varrho}^{3}(\Omega) \times W_{\varrho}^{1}(\Omega) \times W_{\varrho}^{1}(\Omega) \times L_{1}\left(\mathbb{R}^{+}, W_{\varrho}^{1}\left(\Omega, \mathbb{R}^{N}\right)\right) \times L_{1}\left(\mathbb{R}^{+}, W_{\varrho}^{1}\left(\Omega, \mathbb{R}^{N}\right)\right)
$$

with $\partial_{v} f^{0}=0$, there exists a unique global nonnegative solution $(f, m, w, q, p) \in C\left(\mathbb{R}^{+}, X\right)$ to $(1.14)-(1.21)$.

It will be shown that the functions $f, m, w$, and $q$ are much smoother than stated above and are classical solutions to the corresponding equations. The function $p$ also possesses more regularity with respect to the spatial variable and solves equation (1.18) in a mild sense. We refer to Theorem 3.1 for a precise statement of our results.

\section{Auxiliary results: The semilinear case}

In this section we consider the evolution equation (1.22)-(1.23). However, we merely collect the basic properties of the solution and postpone most of the technical proofs to the Appendix.

Throughout this paper $\Omega \subset \mathbb{R}^{n}$ denotes a bounded and smooth domain with $n \leqslant 3$. For $N \in \mathbb{N}$ fixed and $1 \leqslant \varrho \leqslant \infty$ and $\vartheta \geqslant 0$ given, we set

$$
L_{\varrho}:=L_{\varrho}\left(\Omega, \mathbb{R}^{N}\right), \quad W_{\varrho}^{\vartheta}:=W_{\varrho}^{\vartheta}\left(\Omega, \mathbb{R}^{N}\right)
$$


and define

$$
W_{\varrho, \mathscr{B}}^{\vartheta}:= \begin{cases}\left\{u \in W_{\varrho}^{\vartheta} ; \partial_{v} u=0\right\}, & \vartheta>1+1 / \varrho, \\ W_{\varrho}^{\vartheta}, & 0 \leqslant \vartheta<1+1 / \varrho,\end{cases}
$$

where $\partial_{v} u=\left(\partial_{v} u_{1}, \ldots, \partial_{v} u_{N}\right)$ for $u=\left(u_{1}, \ldots, u_{N}\right) \in W_{\varrho}^{\vartheta}$. To account for age structure we similarly put

$$
\mathbb{L}_{\varrho}:=L_{1}\left((0, \infty), L_{\varrho}\right), \quad \mathbb{W}_{\varrho}^{\vartheta}:=L_{1}\left((0, \infty), W_{\varrho}^{\vartheta}\right), \quad \text { and } \quad \mathbb{W}_{\varrho, \mathscr{B}}^{\vartheta}:=L_{1}\left((0, \infty), W_{\varrho, \mathscr{B}}^{\vartheta}\right) .
$$

By $\mathbb{L}_{\varrho}^{+}$we denote the positive cone of $\mathbb{L}_{\varrho}$. In order to keep the notation simple we agree upon the following convention: in the sequel, the letters $f, m$, and $w$ always stand for scalar-valued functions (defined on $\Omega$ ); that is, we write $f \in L_{\varrho}$ etc. for $f \in L_{\varrho}(\Omega, \mathbb{R}$ ) and we shall do the same for $W_{\varrho}^{\vartheta}$ and $W_{\varrho, \mathscr{B}}^{\vartheta}$. Moreover, given $\phi \in \mathbb{L}_{\varrho}$ and $(a, x) \in \mathbb{R}^{+} \times \Omega$ we write $\phi(a, x)$ instead of $\phi(a)(x)$ and we write $\phi(a)$ for $\phi(a, \cdot)$. We use similar notation for other functions depending on two or more arguments hoping that the meaning will be clear from the context.

Let $E$ and $F$ be Banach spaces. We use the notation $\mathscr{L}(E, F)$ for the set of all linear bounded operators from $E$ into $F$ equipped with the usual uniform operator norm. We write $\mathscr{L}(E)$ if $E=F$. If $E$ is dense in $F$ we denote by $\mathscr{H}(E, F)$ the subset of $\mathscr{L}(E, F)$ consisting of all negative generators of analytic semigroups on $F$ with domain $E$. Moreover, $A \in \mathscr{H}^{+}(E, F)$ means that $-A$ is resolvent positive (cf. [3]). We write $C_{b}^{1-}(E, F)$ for the set of all functions from $E$ into $F$ which are uniformly Lipschitz continuous on bounded subsets of $E$. If $B \subset E$ we write $g \in C^{k-}(B, F)$ provided the $(k-1)$ th-derivative of $g$ is Lipschitz continuous.

For $T>0$ let $\Delta_{T}:=\left\{(t, s) \in J_{T} \times J_{T} ; 0 \leqslant s \leqslant t \leqslant T\right\}$ and $\Delta_{T}^{*}:=\left\{(t, s) \in \Delta_{T} ; s<t\right\}$, where $J_{T}:=[0, T]$.

\subsection{The age-diffusion evolution operator}

We study the abstract version of the equation (1.18). More precisely, given $\phi \in \mathbb{L}_{\varrho}$, $0 \leqslant s<T$, and $g \in C\left([0, T], L_{\varrho}\right)$ we seek a (generalized) solution $u=u(t, a)$ in $L_{\varrho}$ to the problem

$$
\begin{aligned}
\partial_{t} u+\partial_{a} u+A(t) u & =g(t), & & a>0, s<t \\
u(t, 0) & =2 \int_{0}^{\infty} \mathfrak{b}(t, a) u(t, a) \mathrm{d} a, & & s<t \leqslant T, \\
u(s, a) & =\phi(a), & & a>0 .
\end{aligned}
$$

We first consider the homogeneous equation with $g \equiv 0$. Let $\varrho \in(1, \infty)$ and suppose that $A=A(t)$ generates an evolution system $\left\{U_{A}(t, s) ;(t, s) \in \Delta_{T}\right\}$ on $L_{\varrho}$. We may solve then $(P)_{s, \phi, 0}$ formally along characteristics. Writing $[\mathscr{U}(t, s) \phi](a)$ for the solution instead of $u(t, a)$, one obtains the formula

$$
[\mathscr{U}(t, s) \phi](a):= \begin{cases}U_{A}(t, t-a)(B \phi)(t-a, s), & 0 \leqslant a \leqslant t-s, \\ U_{A}(t, s) \phi(a-t+s), & 0 \leqslant t-s<a,\end{cases}
$$


where the function $B \phi$ satisfies the Volterra equation

$$
\begin{aligned}
(B \phi)(t, s)= & 2 \int_{0}^{t-s} \mathfrak{b}(t, a) U_{A}(t, t-a)(B \phi)(t-a, s) \mathrm{d} a \\
& +2 \int_{t-s}^{\infty} \mathfrak{b}(t, a) U_{A}(t, s) \phi(a-t+s) \mathrm{d} a .
\end{aligned}
$$

In order to emphasize the dependence of $\mathscr{U}$ and $B \phi$ on the data $A$ and $\mathfrak{b}$, respectively, we sometimes write $\mathscr{U}_{[A, \mathfrak{b}]}$ and $B_{[A, \mathfrak{b}]} \phi$ instead. In Appendix we will prove that the Volterra equation (2.2) has a unique solution $B \phi \in C\left(\Delta_{T}, L_{\varrho}\right)$ so that we indeed may define $\mathscr{U}$ by way of (2.1). Notice that from (2.1)-(2.2)

$$
(B \phi)(t, s)=2 \int_{0}^{\infty} \mathfrak{b}(t, a)[\mathscr{U}(t, s) \phi](a) \mathrm{d} a=[\mathscr{U}(t, s) \phi](0), \quad(t, s) \in \Delta_{T} .
$$

We then have the following fundamental result which shows that (2.1) defines an evolution operator that depends Lipschitz continuously on the data $A$ and $\mathfrak{b}$ :

Proposition 2.1 Fix numbers $\varrho \in(1 \vee n / 2, \infty), 2 \eta \in(n / \varrho, 2) \backslash\{1+1 / \varrho\}, \rho>0, M>0$, and $T>0$. Suppose that

$$
A \in C^{\rho}\left(J_{T}, \mathscr{H}^{+}\left(W_{\varrho, \mathscr{B}}^{2}, L_{\varrho}\right)\right) \quad \text { with } \quad\|A\|_{C^{\rho}\left(J_{T}, \mathscr{H}\left(W_{\varrho, \mathscr{B}}^{2}, L_{\varrho}\right)\right)} \leqslant M
$$

and denote by $\left\{U_{A}(t, s) ;(t, s) \in \Delta_{T}\right\}$ the positive evolution operator on $L_{\varrho}$ generated by $-A$. Further suppose that

$$
\mathfrak{b} \in F_{\eta}:=C\left(J_{T}, L_{\infty}^{+}\left((0, \infty),\left(W_{\varrho, \mathscr{B}}^{2 \eta}(\Omega)\right)^{N \times N}\right)\right) \quad \text { with } \quad\|\mathfrak{b}\|_{F_{\eta}} \leqslant M .
$$

Defining $\mathscr{U}(t, s)=\mathscr{U}_{[A, \mathfrak{b}]}(t, s)$ by way of $(2.1),\left\{\mathscr{U}(t, s) ;(t, s) \in \Delta_{T}\right\}$ is a positive evolution operator on $\mathbb{L}_{\varrho}$, that is:

(i) $\mathscr{U}(t, s) \in \mathscr{L}\left(\mathbb{L}_{\varrho}\right)$ leaves $\mathbb{L}_{\varrho}^{+}$invariant for $(t, s) \in \Delta_{T}$,

(ii) $\mathscr{U}(t, t) \phi=\phi$ for $\phi \in \mathbb{L}_{\varrho}$ and $t \in J_{T}$,

(iii) $\mathscr{U}(t, r) \mathscr{U}(r, s)=\mathscr{U}(t, s)$ for $0 \leqslant s \leqslant r \leqslant t \leqslant T$,

(iv) $[(t, s) \mapsto \mathscr{U}(t, s) \phi] \in C\left(\Delta_{T}, \mathbb{W}_{\varrho, \mathscr{B}}^{2 \theta}\right)$ for $\phi \in \mathbb{W}_{\varrho, \mathscr{B}}^{2 \theta}$ and $2 \theta \in[0,2) \backslash\{1+1 / \varrho\}$.

Moreover,

$$
\|\mathscr{U}(t, s)\|_{\mathscr{L}\left(\mathbb{W}_{Q, \mathscr{B}}^{2 \theta}, \mathbb{W}_{\varrho, \mathscr{B}}^{2 \sigma}\right)} \leqslant c_{T}(M)(t-s)^{\bar{\theta}-\sigma}, \quad(t, s) \in \Delta_{T}^{*}
$$

provided $0 \leqslant \bar{\theta} \leqslant \theta \leqslant \sigma \leqslant 1$ with $2 \theta, 2 \sigma \neq 1+1 / \varrho$ and $\bar{\theta}<\theta$ if $0<\theta<\sigma<1$. Finally, the evolution system $\mathscr{U}=\mathscr{U}_{[A, \mathfrak{b}]}$ depends Lipschitz continuously on the data $A$ and $\mathfrak{b}$ in the following sense: If $A$ and $\bar{A}$ both satisfy (2.4) and if $\mathfrak{b}$ and $\overline{\mathfrak{b}}$ both satisfy (2.5), then, defining

$$
\|A-\bar{A}\| \mid:=\max _{t \in J_{T}}\|A(t)-\bar{A}(t)\|_{\mathscr{L}\left(W_{Q, O B}^{2}, L_{\varrho}\right)}
$$


we have

$$
\left\|\mathscr{U}_{[A, \mathfrak{b}]}(t, s)-\mathscr{U}_{[\bar{A}, \overline{\mathfrak{b}}]}(t, s)\right\|_{\mathscr{L}\left(\mathbb{W}_{\varrho, g}^{2 \theta}, \mathbb{W}_{\varrho, g}^{2 \sigma}\right)} \leqslant c_{T}(M)(t-s)^{\theta-\sigma}\left[\|\mathfrak{b}-\overline{\mathfrak{b}}\|_{F_{\eta}}+\|A-\bar{A}\|\right]
$$

for $(t, s) \in \Delta_{T}^{*}$ provided that $2 \theta \in(0,2] \backslash\{1+1 / \varrho\}$ and $2 \sigma \in[0,2) \backslash\{1+1 / \varrho\}$.

The constant $c_{T}(M)$ denotes, in the statement above but also in the following, a generic constant which depends increasingly on the numbers $T$ and $M$ but not on other relevant variables.

We postpone the proof of Proposition 2.1 to the Appendix in order not to further delay our main result on the global well-posedness of (1.1)-(1.8).

The existence of the evolution system $\mathscr{U}=\mathscr{U}_{[A, \mathfrak{b}]}$ now yields the basis by which to define a meaningful mild solution to $(P)_{s, \phi, g}$ provided $A$ and $\mathfrak{b}$ satisfy the assumptions of Proposition 2.1.

Definition 2.2 Let $A$ and $\mathfrak{b}$ satisfy (2.4) and (2.5), respectively, and let $\mathscr{U}=\mathscr{U}_{[A, \mathfrak{b}]}$ denote the evolution operator provided by Proposition 2.1. For $\phi \in \mathbb{L}_{\varrho}$ and $g \in C\left(J_{T}, \mathbb{L}_{\varrho}\right)$, the mild solution $u \in C\left([s, T], \mathbb{L}_{\varrho}\right)$ of $(P)_{s, \phi, g}$ is defined by

$$
u(t):=\mathscr{U}(t, s) \phi+\int_{s}^{t} \mathscr{U}(t, \sigma) g(\sigma) \mathrm{d} \sigma, \quad s \leqslant t \leqslant T .
$$

We also postpone a further justification of this definition to the Appendix. In particular we will show there that classical solutions of $(P)_{s, \phi, g}$ are necessarily mild solutions in the sense just defined.

The following lemma states that integrating the mild solution with respect to $a$ indeed yields the solution to the corresponding integrated version of the first equation in $(P)_{s, \phi, g}$.

Lemma 2.3 Suppose $A$ and $\mathfrak{b}$ satisfy (2.4), (2.5) and let $\mathscr{U}=\mathscr{U}_{[A, \mathfrak{b}]}$. Given $\phi \in \mathbb{L}_{\varrho}$ and $g \in C\left(J_{T}, \mathbb{L}_{\varrho}\right)$ let

$$
p(t):=\mathscr{U}(t, 0) \phi+\int_{0}^{t} \mathscr{U}(t, s) g(s) \mathrm{d} s \quad \text { and } \quad P(t):=\int_{0}^{\infty} p(t, a) \mathrm{d} a, \quad t \in J_{T} .
$$

Then $P \in C\left(J_{T}, L_{\varrho}\right)$ satisfies, for $t \in J_{T}$,

$$
P(t)=U_{A}(t, 0) \int_{0}^{\infty} \phi(a) \mathrm{d} a+\int_{0}^{t} U_{A}(t, s)\left\{\int_{0}^{\infty} g(s, a) \mathrm{d} a+2 \int_{0}^{\infty} \mathfrak{b}(s, a) p(s, a) \mathrm{d} a\right\} \mathrm{d} s .
$$

Proof Let $t \in J_{T}$. Integrating $p(t)$ with respect to $a \in(0, \infty)$ and using (2.1) we derive

$$
\begin{aligned}
P(t)= & \int_{0}^{t} U_{A}(t, a)(B \phi)(a, 0) \mathrm{d} a+U_{A}(t, 0) \int_{0}^{\infty} \phi(a) \mathrm{d} a \\
& +\int_{0}^{t} U_{A}(t, a) \int_{0}^{a}(B g(r))(a, r) \mathrm{d} r \mathrm{~d} a+\int_{0}^{t} U_{A}(t, a) \int_{0}^{\infty} g(a, r) \mathrm{d} r \mathrm{~d} a .
\end{aligned}
$$


Noticing that (2.3) ensures

$$
2 \int_{0}^{\infty} \mathfrak{b}(t, a) p(t, a) \mathrm{d} a=(B \phi)(t, 0)+\int_{0}^{t}(B g(s))(t, s) \mathrm{d} s
$$

the assertion follows.

We will use the next lemma later in order to prove positivity of solutions to (1.17), (1.18)

Lemma 2.4 Let $A$, $\mathfrak{b}$ satisfy (2.4), (2.5) and $\mathscr{U}=\mathscr{U}_{[A, \mathfrak{b}]}$. Let $G \in C^{\rho}\left(J_{T}, \mathscr{H}\left(\mathbb{W}_{\varrho, \mathscr{B}}^{2}, \mathbb{L}_{\varrho}\right)\right)$ generate a positive evolution operator $\left\{U_{G}(t, s) ;(t, s) \in \Delta_{T}\right\}$, on $\mathbb{L}_{\varrho}$. Further suppose that $H_{j} \in C\left(J_{T}, L_{\infty}\left(\mathbb{R}^{+}, L_{\infty}(\Omega)^{N \times N}\right)\right), j=1,2,3,4$, with $H_{j} \geqslant 0$ for $j=2,3$. Define

$$
\mathscr{V}(t, s):=\left[\begin{array}{cc}
U_{G}(t, s) & 0 \\
0 & \mathscr{U}(t, s)
\end{array}\right], \quad H(t):=\left[\begin{array}{ll}
H_{1}(t) & H_{2}(t) \\
H_{3}(t) & H_{4}(t)
\end{array}\right]
$$

for $(t, s) \in \Delta_{T}$. Then, for any given $u^{0} \in \mathbb{L}_{\varrho}^{+} \times \mathbb{L}_{\varrho}^{+}$, there exists a unique solution $u \in C\left([0, T], \mathbb{L}_{\varrho}^{+} \times \mathbb{L}_{\varrho}^{+}\right)$to

$$
u(t)=\mathscr{V}(t, 0) u^{0}+\int_{0}^{t} \mathscr{V}(t, s) H(s) u(s) \mathrm{d} s, \quad t \in J_{T} .
$$

Proof Let $\mathbb{E}:=\mathbb{L}_{\varrho} \times \mathbb{L}_{\varrho}$ and $\mathbb{E}^{+}:=\mathbb{L}_{\varrho}^{+} \times \mathbb{L}_{\varrho}^{+}$. Put $\omega:=\max \left\{\left\|H_{1}\right\|_{\infty},\left\|H_{4}\right\|_{\infty}\right\}$ and observe that $H_{\omega}(t) \in \mathscr{L}(\mathbb{E})$ with $H_{\omega}(t)\left(\mathbb{E}^{+}\right) \subset \mathbb{E}^{+}$for $t \in J_{T}$, where

$$
H_{\omega}(t):=\left[\begin{array}{cc}
H_{1}(t)+\omega & H_{2}(t) \\
H_{3}(t) & H_{4}(t)+\omega
\end{array}\right], \quad t \in J_{T} .
$$

Writing $\mathscr{V}_{\omega}$ for the operator obtained by replacing $G$ and $A$ by $G+\omega$ and $A+\omega$, respectively, it is standard to show that there is a unique solution $u \in C\left([0, T], \mathbb{E}^{+}\right)$to

$$
u(t)=\mathscr{V}_{\omega}(t, 0) u^{0}+\int_{0}^{t} \mathscr{V}_{\omega}(t, s) H_{\omega}(s) u(s) \mathrm{d} s, \quad t \in J_{T} .
$$

Lemma A.3 in Appendix implies that $u$ solves (2.8) as well.

\subsection{Further auxiliary results}

We state some auxiliary results required in the next section. Let $E$ be a Banach space and $T>0$. Given $\mu \in \mathbb{R}$, we denote by $B C_{\mu}((0, T], E)$ the Banach space of all functions $u:(0, T] \rightarrow E$ such that $\left(t \mapsto t^{\mu} u(t)\right)$ is bounded and continuous from $(0, T]$ into $E$, equipped with the norm

$$
u \mapsto\|u\|_{C_{\mu}((0, T], E)}:=\sup _{t \in(0, T]} t^{\mu}\|u(t)\|_{E} .
$$

We write $C_{\mu}((0, T], E)$ for the closed linear subspace thereof consisting of all $u$ satisfying $t^{\mu} u(t) \rightarrow 0$ in $E$ as $t \rightarrow 0^{+}$. Observe the embeddings $C_{0}((0, T], E) \hookrightarrow C([0, T], E)$ and $C_{v}((0, T], E) \hookrightarrow C_{\mu}((0, T], E)$ for $v \leqslant \mu$. 
Lemma 2.5 Let $\varrho \in(1, \infty)$. Given $\alpha \in C^{1}(\bar{\Omega})$ with $\alpha>0$ on $\bar{\Omega}$, let $\left\{e^{-t A_{\alpha}} ; t \geqslant 0\right\}$ denote the analytic semigroup on $L_{\varrho}$ generated by

$$
-A_{\alpha} m:=\nabla_{x} \cdot\left(\alpha(\cdot) \nabla_{x} m\right), \quad m \in D\left(A_{\alpha}\right):=W_{\varrho, \mathscr{B}}^{2}
$$

If $T>0$ and $2 v \leqslant 2 \leqslant 2 \xi<2+2 v$ with $2 v \neq 1+1 / \varrho$, then

$$
\left[t \mapsto e^{-t A_{\alpha}} m^{0}\right] \in C_{\xi-v}\left((0, T], W_{\varrho, \mathscr{B}}^{2 \xi}\right), \quad m^{0} \in W_{\varrho, \mathscr{B}}^{2 v} .
$$

Proof Denoting by $D\left(A_{\alpha}^{2}\right)$ the domain of $A_{\alpha}^{2}$ (equipped with the graph norm), it follows from [28, Thm.5.4.1, Thm.5.3.4] that $D\left(A_{\alpha}^{2}\right)$ is continuously embedded in $W_{\varrho, \mathscr{B}}^{4}$. Therefore,

$$
\left(D\left(A_{\alpha}\right), D\left(A_{\alpha}^{2}\right)\right)_{\theta, \varrho} \hookrightarrow W_{\varrho, \mathscr{B}}^{2+2 \theta}, \quad \theta \in(0,1) \backslash\{1 / 2\},
$$

where $(\cdot, \cdot)_{\theta, \varrho}$ denotes the real interpolation functor, whence

$$
\left\|e^{-t A_{\alpha}}\right\|_{\mathscr{L}\left(W_{Q, \mathscr{B}}^{2 v}, W_{Q, B}^{2 \xi}\right)} \leqslant c(T) t^{v-\xi}, \quad 0<t \leqslant T
$$

by [3, V.Thm.2.1.3]. Now the statement follows analogously to [4, Prop.6].

Clearly, given functions $m$ and $k$, the solution $f$ to equation (1.1) is

$$
f(t)=e^{-k \int_{0}^{t} m(\sigma) \mathrm{d} \sigma} f^{0}
$$

We then have:

Lemma 2.6 Let $T>0$ and $M>0$. Suppose that $\varrho \in(1 \vee n / 2, \infty), 0 \leqslant r<v$, and $0 \leqslant \mu<1-\varepsilon \leqslant 1$. Given $k \in W_{\varrho, \mathscr{B}}^{2+2 v}$ and $f^{0} \in W_{\varrho, \mathscr{B}}^{2+2 v}$ set

$$
\zeta(m)(t):=e^{-k \int_{0}^{t} m(\sigma) \mathrm{d} \sigma} f^{0} .
$$

Then $\zeta \in C_{b}^{1-}\left(C_{\mu}\left((0, T], W_{\varrho, \mathscr{B}}^{2+2 v}\right), C^{1-\mu-\varepsilon}\left([0, T], W_{\varrho, \mathscr{B}}^{2+2 r}\right)\right)$. More precisely,

$$
\|\zeta(m)-\zeta(\bar{m})\|_{C^{1-\mu-\varepsilon}\left([0, T], W_{\varrho, \mathscr{B}}^{2+2 r}\right)} \leqslant c_{T}(M) T^{\varepsilon}\|m-\bar{m}\|_{C_{\mu}\left((0, T], W_{Q, \mathscr{B}}^{2+2 v}\right)}
$$

whenever $\left\|f^{0}\right\|_{W_{\varrho, \mathscr{B}}^{2+2 v}} \leqslant M,\|m\|_{C_{\mu}\left((0, T], W_{Q, \overparen{B}}^{2+2 v}\right)} \leqslant M$, and $\|\bar{m}\|_{C_{\mu}\left((0, T], W_{Q, \mathscr{B}}^{2+2 v}\right)} \leqslant M$.

Proof Analogously to [30, Lem.2.1] it follows from [2, Thm.4.1] that

$$
\|\zeta(m)(t)-\zeta(\bar{m})(t)\|_{W_{Q, \mathscr{B}}^{2+2 r}} \leqslant c_{T}(M) t^{1-\mu}\|m-\bar{m}\|_{C_{\mu}\left((0, T], W_{Q, B}^{2+2 v}\right)}, \quad t \in[0, T] .
$$

Writing

$$
\partial_{t}(\zeta(m)-\zeta(\bar{m}))(t)=-k(m-\bar{m})(t) \zeta(m)-k \bar{m}(t)(\zeta(m)-\zeta(\bar{m}))(t)
$$


and using the fact that pointwise multiplication $W_{\varrho}^{2+2 r} \times W_{\varrho}^{2+2 r} \rightarrow W_{\varrho}^{2+2 r}$ is continuous (cf. [2, Thm.4.1]), we derive that, for $0 \leqslant s \leqslant t \leqslant T$,

$$
\begin{aligned}
& \|(\zeta(m)-\zeta(\bar{m}))(t)-(\zeta(m)-\zeta(\bar{m}))(s)\|_{W_{e, g}^{2+2 r}} \\
& \leqslant \int_{s}^{t}\left\|\partial_{\sigma}(\zeta(m)-\zeta(\bar{m}))(\sigma)\right\|_{W_{\varrho, B}^{2+2 r}} \mathrm{~d} \sigma \\
& \leqslant c_{T}(M) t^{\varepsilon} \int_{S}^{t} \sigma^{-\mu-\varepsilon} \sigma^{\mu}\|m(\sigma)-\bar{m}(\sigma)\|_{W_{Q, B}^{2+2 r}}\|\zeta(m)(\sigma)\|_{W_{Q, B}^{2+2 r}} \mathrm{~d} \sigma \\
& +c_{T}(M) t^{\varepsilon} \int_{S}^{t} \sigma^{-\mu-\varepsilon} \sigma^{\mu}\|\bar{m}(\sigma)\|_{W_{\varrho, \mathscr{B}}^{2+2 r}}\|\zeta(m)(\sigma)-\zeta(\bar{m})(\sigma)\|_{W_{\varrho, \mathscr{B}}^{2+2 r}} \mathrm{~d} \sigma \\
& \leqslant c_{T}(M) T^{\varepsilon}\left(t^{1-\mu-\varepsilon}-s^{1-\mu-\varepsilon}\right)\|m-\bar{m}\|_{C_{\mu}\left((0, T], W_{\varrho, \mathscr{B}}^{2+2 v}\right)},
\end{aligned}
$$

from which the assertion follows.

We conclude this section with the following result about Nemitskii operators.

Lemma 2.7 Let $\varrho \in(n, \infty), \xi \in(n / \varrho, 2) \backslash\{1+1 / \varrho\}, \bar{\xi} \in(0, \xi) \backslash\{1+1 / \varrho\}$ and $l \in \mathbb{N}$. Given $\mathfrak{f} \in C^{3-}\left(\mathbb{R}^{l}\right)$ put $\mathfrak{F}(u)(x):=\mathfrak{f}(u(x))$ for $x \in \Omega$ and $u: \Omega \rightarrow \mathbb{R}^{l}$. Then we have $\mathfrak{F} \in C_{b}^{1-}\left(\left(W_{\varrho, \mathscr{B}}^{\xi}\right)^{l}, W_{\varrho, \mathscr{B}}^{\xi}\right)$.

Proof We may assume that $l=1$. Fix $R>0$.

(i) First let $\xi \in(n / \varrho, 1)$. Owing to $\|\cdot\|_{\infty} \leqslant c_{0}\|\cdot\|_{W_{\varrho, \mathscr{B}}^{\xi}}$ (since $\left.W_{\varrho, \mathscr{B}}^{\xi} \hookrightarrow L_{\infty}\right)$, and

$$
\left|\mathfrak{f}^{\prime}(r)\right| \leqslant c(R), \quad\left|\mathfrak{f}^{\prime}(r)-\mathfrak{f}^{\prime}(s)\right| \leqslant c(R)|r-s|, \quad|r|,|s| \leqslant c_{0} R
$$

for some constant $c(R)>0$, it follows from the mean value theorem that, for $\|u\|_{W_{\varrho, g}^{\xi}} \leqslant R$, $\|\bar{u}\|_{W_{\varrho, Q}^{\xi}} \leqslant R$, and $x, y \in \Omega$,

$$
\begin{aligned}
& |(\mathfrak{F}(u)-\mathfrak{F}(\bar{u}))(x)-(\mathfrak{F}(u)-\mathfrak{F}(\bar{u}))(y)| \\
& \quad \leqslant\left|\int_{0}^{1} \mathfrak{f}^{\prime}(u(x)+\sigma[\bar{u}(x)-u(x)]) \mathrm{d} \sigma(u(x)-\bar{u}(x)-u(y)+\bar{u}(y))\right| \\
& \quad+\left|\int_{0}^{1}\left\{\mathfrak{f}^{\prime}(u(x)+\sigma[\bar{u}(x)-u(x)])-\mathfrak{f}^{\prime}(u(y)+\sigma[\bar{u}(y)-u(y)])\right\} \mathrm{d} \sigma\right||u(y)-\bar{u}(y)| \\
& \quad \leqslant c(R)\left\{|u(x)-\bar{u}(x)-u(y)+\bar{u}(y)|+[|u(x)-u(y)|+|\bar{u}(x)-\bar{u}(y)|]\|u-\bar{u}\|_{\infty}\right\} .
\end{aligned}
$$

Therefore,

$$
\begin{aligned}
\|\mathfrak{F}(u)-\mathfrak{F}(\bar{u})\|_{W_{\varrho, \mathscr{B}}^{\xi}}^{\varrho} \leqslant & \|\mathfrak{F}(u)-\mathfrak{F}(\bar{u})\|_{L_{\varrho}}^{\varrho} \\
& +\int_{\Omega \times \Omega} \frac{\mid(\mathfrak{F}(u)-\mathfrak{F}(\bar{u}))(x)-(\mathfrak{F}(u)-\mathfrak{F}(\bar{u}))(y))\left.\right|^{\varrho}}{|x-y|^{n+\xi} \varrho} \mathrm{d}(x, y) \\
\leqslant & c(R)\|u-\bar{u}\|_{W_{\varrho, \mathscr{B}}^{\xi}}^{\varrho}+c(R)\|u-\bar{u}\|_{L_{\infty}}^{\varrho},
\end{aligned}
$$

whence $\mathfrak{F} \in C_{b}^{1-}\left(W_{\varrho, \mathscr{B}}^{\xi}, W_{\varrho, \mathscr{B}}^{\xi}\right)$. Note that we only used $\mathfrak{f} \in C^{2-}$. 
(ii) Now let $\xi \in(1,2) \backslash\{1+1 / \varrho\}$ and $\bar{\xi} \in(1, \xi) \backslash\{1+1 / \varrho\}$. Choose $\tau \in(n / \varrho, 1)$ with $\tau>\bar{\xi}-1$. Then pointwise multiplication $W_{\varrho}^{\tau} \times W_{\varrho}^{\xi-1} \rightarrow W_{\varrho}^{\bar{\xi}-1}$ is continuous, see [2, Thm.4.1]. Therefore,

$$
\begin{aligned}
\|\mathfrak{F}(u)-\mathfrak{F}(\bar{u})\|_{W_{\varrho}^{\bar{\xi}}} \leqslant & \|\mathfrak{F}(u)-\mathfrak{F}(\bar{u})\|_{L_{\varrho}}+\sum_{j=1}^{n}\left\|\left(\mathfrak{f}^{\prime}(u)-\mathfrak{f}^{\prime}(\bar{u})\right) \partial_{j} u\right\|_{W_{\varrho}^{\bar{\xi}}-1} \\
& +\sum_{j=1}^{n}\left\|\mathfrak{f}^{\prime}(u) \partial_{j}(u-\bar{u})\right\|_{W_{\varrho}^{\bar{\xi}}-1} \\
\leqslant & c(R)\|u-\bar{u}\|_{L_{\varrho}}+\left\|\mathfrak{f}^{\prime}(u)-\mathfrak{f}^{\prime}(\bar{u})\right\|_{W_{\varrho}^{\tau}}\|u\|_{W_{\varrho}^{\xi}} \\
& +\left\|\mathfrak{f}^{\prime}(\bar{u})\right\|_{W_{\varrho}^{\tau}}\|u-\bar{u}\|_{W_{\varrho}^{\xi}}
\end{aligned}
$$

for $\|u\|_{W_{\varrho}^{\xi}},\|\bar{u}\|_{W_{\varrho}^{\xi}} \leqslant R$, and thus

$$
\|\mathfrak{F}(u)-\mathfrak{F}(\bar{u})\|_{W_{\varrho}^{\bar{\xi}}} \leqslant c(R)\|u-\bar{u}\|_{W_{\varrho}^{\xi}}
$$

follows from part (i) since $\tau \in(n / \varrho, 1), W_{\varrho}^{\xi} \hookrightarrow W_{\varrho}^{\tau}$, and $\mathfrak{f}^{\prime} \in C^{2-}$. The case $\xi=1$ is obvious.

\section{Global well-posedness}

We are now in a position to prove global existence and uniqueness of nonnegative solutions to equations (1.14)-(1.21). To do so we require the following assumptions to hold:

$\left(A_{1}\right) \alpha, \beta \in C^{1}(\bar{\Omega})$ with $\alpha(x), \beta(x)>0$ for $x \in \bar{\Omega} ; \gamma, \delta \in C^{2-}\left(\mathbb{R}^{+}, \mathbb{R}^{N}\right)$ with $\gamma(z), \delta(z)>0$ for $z \geqslant 0$;

$\left(A_{2}\right)$ there is some $\epsilon>0$ such that $h \in C^{4 \epsilon}(\bar{\Omega}), k \in W_{\varrho, \mathscr{B}}^{2+3 \epsilon}(\Omega), e \in L_{\infty}(\Omega)$, and $h, k, e$ are nonnegative;

$\left(A_{3}\right) \chi \in C^{3-}\left(\mathbb{R}^{+}, \mathbb{R}^{N}\right)$ is nonnegative;

$\left(A_{4}\right) \Theta \in C^{3-}\left(\mathbb{R}^{2 N}\right)$ is nonnegative;

$\left(A_{5}\right) \Gamma \in C^{0,1-}\left(\bar{\Omega} \times \mathbb{R}^{+}\right)$is nonnegative;

$\left(A_{6}\right) \Lambda \in C^{0,1-}\left(\bar{\Omega} \times \mathbb{R}^{2 N}\right)$ is nonnegative;

$\left(A_{7}\right)$ each $\Xi \in\{b, \Sigma, \Phi, \Psi, r\}$ belongs to $C^{0,2}\left(\mathbb{R}^{+} \times \mathbb{R}^{1+2 N}\right)$ and is nonnegative and for $R>0$ there exists $c(R)>0$ such that

$$
\left|D_{2}^{i} \Xi(a, z)-D_{2}^{i} \Xi(a, \bar{z})\right| \leqslant c(R)|z-\bar{z}|, \quad a>0, \quad z, \bar{z} \in \mathbb{R}^{1+2 N}, \quad i=0,1,2 ;
$$

$\left(A_{8}\right) \psi=\left(\psi_{j, l}\right) \in C^{0,2}\left(\mathbb{R}^{+} \times \mathbb{R}^{1+2 N}, \mathbb{R}^{N \times N}\right)$ is nonnegative; for $R>0$ there exists $c(R)>0$ such that

$$
\left|D_{2}^{i} \psi(a, z)-D_{2}^{i} \psi(a, \bar{z})\right| \leqslant c(R)|z-\bar{z}|, \quad a>0, \quad z, \bar{z} \in \mathbb{R}^{1+2 N}, \quad i=0,1,2,
$$

and $\psi$ satisfies the additional constraint $\sum_{l=1}^{N} \psi_{j, l}=1$ for $j=1, \ldots, N$. 
In order to prove that the solutions exist globally we will assume in addition that $\left(A_{9}\right)$ there exists $\kappa \in C\left(\mathbb{R}^{+}\right)$such that

$$
\Xi_{j}(a, w, P, Q) \leqslant \kappa(w), \quad a>0, \quad(w, P, Q) \in \mathbb{R} \times \mathbb{R}^{N} \times \mathbb{R}^{N}, \quad j=1, \ldots, N,
$$

for $\Xi \in\{b, \psi, \Sigma, \Phi, \Psi, \Upsilon\}$ and also

$$
\psi_{j, l}(a, w, P, Q) \leqslant \kappa(w), \quad a>0, \quad(w, P, Q) \in \mathbb{R} \times \mathbb{R}^{N} \times \mathbb{R}^{N}, \quad j, l=1, \ldots, N
$$

$\left(A_{10}\right)$ there exists $c_{\Theta}>0$ such that $\Theta(P, Q) \leqslant c_{\Theta}\left(1+\sum_{j=1}^{N}\left(P_{j}+Q_{j}\right)\right)$ for $P, Q \in \mathbb{R}^{N}$ with $P, Q \geqslant 0$.

Here and in the following we mean by $z \geqslant 0$ for $z=\left(z_{1}, \ldots, z_{N}\right) \in \mathbb{R}^{N}$ that $z_{j} \geqslant 0$ for all $j \in\{1, \ldots, N\}$. Moreover, $g \in C^{0,1-}$ means that $g$ is jointly continuous with respect to both arguments and Lipschitz continuous with respect to the second argument. For $b$ and $\psi$ as in $\left(A_{7}\right)$ and $\left(A_{8}\right)$, respectively, we define $\mathfrak{b}$ by (1.13) and we use the matrix notation for $\Sigma, \Psi, \Phi, \Upsilon$ and the notation for the differential operators in divergence form as in (1.9)-(1.12).

Our main result regarding global existence and uniqueness of nonnegative solutions to (1.14)-(1.21) reads as follows:

Theorem 3.1 Suppose $\left(A_{1}\right)-\left(A_{8}\right)$ and let $\varrho \in(n, \infty)$. Given $2 \xi \in(n / \varrho, 2) \backslash\{1+1 / \varrho\}, 2 \lambda \in$ $(0,2) \backslash\{1+1 / \varrho\}$, and any nonnegative initial value

$$
\left(f^{0}, m^{0}, w^{0}, q^{0}, p^{0}\right) \in X:=W_{\varrho, \mathscr{B}}^{2+\epsilon} \times W_{\varrho, \mathscr{B}}^{2 \lambda} \times W_{\varrho, \mathscr{B}}^{2 \xi} \times \mathbb{W}_{\varrho, \mathscr{B}}^{2 \xi} \times \mathbb{W}_{\varrho, \mathscr{B}}^{2 \xi}
$$

there exists a unique maximal nonnegative solution $(f, m, w, q, p) \in C(J, X)$ to (1.14)-(1.21) such that

$$
f \in C^{1}\left(\dot{J}, W_{\varrho, \mathscr{B}}^{2}\right), \quad m, w \in C\left(\dot{J}, W_{\varrho, \mathscr{B}}^{2}\right) \cap C^{1}\left(\dot{J}, L_{\varrho}\right), \quad \text { and } \quad q \in C\left(\dot{J}, \mathbb{W}_{\varrho, \mathscr{B}}^{2}\right) \cap C^{1}\left(\dot{J}, \mathbb{L}_{\varrho}\right),
$$

where $J$ denotes the maximal interval of existence and $\dot{J}:=J \backslash\{0\}$. Here, $f, m, w$, and $q$ are classical solutions to the corresponding equations, while $p$ is a mild solution (in the sense defined in Section A.2). Moreover,

$$
P:=\int_{0}^{\infty} p(\cdot, a, \cdot) \mathrm{d} a \in C\left(\dot{J}, W_{\varrho, \mathscr{B}}^{2}\right) \cap C^{1}\left(\dot{J}, L_{\varrho}\right) .
$$

Finally, if also $\left(A_{9}\right)$ and $\left(A_{10}\right)$ hold, then the solution exists globally, that is, $J=\mathbb{R}^{+}$.

Remark 3.2 It is possible to consider diffusion coefficients $\gamma$ and $\delta$ that, in addition, depend smoothly on the tumour densities $P$ and $Q$, respectively. Local existence of nonnegative unique solutions with the same regularity properties as stated in the above theorem can be shown provided that $2 \xi \in(1+n / \varrho, 2) \backslash\{1+1 / \varrho\}$ and $2 \lambda \in(0,2) \backslash\{1+1 / \varrho\}$. However, our method does not necessarily yield global solutions in this case. 


\subsection{Proof of theorem 3.1: Local existence}

Suppose $\left(A_{1}\right)-\left(A_{8}\right)$ hold. Given $\xi$ and $\lambda$ as in the statement of Theorem 3.1 and $\epsilon$ as in assumption $\left(A_{2}\right)$, choose numbers such that

$$
\frac{n}{\varrho}<2 \bar{\eta}<2 \eta<2 \xi, \quad \mu \in(0,1), \quad 0<2 v<2 \bar{v}<(\epsilon \wedge(2 \mu+2 \lambda-2) \wedge 2 \eta) .
$$

We fix $T, R, R_{0}>0$ and denote by $c(T), c(T, R)$ etc. constants that depend increasingly on $T$ and $R$ etc. For $J_{T}:=[0, T]$ and $\dot{J}_{T}:=(0, T]$ we define the Banach spaces $E_{j}$ by

$$
\begin{aligned}
& E_{1}:=C_{\mu}\left(\dot{J}_{T}, W_{\varrho, \mathscr{B}}^{2+2 v}\right) \cap C\left(J_{T}, W_{\varrho, \mathscr{B}}^{2 \lambda}\right), \\
& E_{2}:=C\left(J_{T}, W_{\varrho, \mathscr{B}}^{2 \eta}\right), \quad E_{3}:=C\left(J_{T}, \mathbb{W}_{\varrho, \mathscr{B}}^{2 \eta}\right)
\end{aligned}
$$

and we set $\mathbb{E}:=\mathbb{E}_{T}:=E_{1} \times E_{2} \times E_{3} \times E_{3}$. Lemma 2.6 then gives that

$$
\zeta \in C_{b}^{1-}\left(E_{1}, C^{1-\bar{\mu}}\left(J_{T}, W_{\varrho, \mathscr{B}}^{2+2 r}\right)\right) \quad \text { for } \quad \zeta(m)(t):=e^{-k \int_{0}^{t} m(\sigma) \mathrm{d} \sigma} f^{0}
$$

where $r \in(0, v)$ and $\bar{\mu} \in(\mu, 1)$. Let $u=(m, w, q, p)$ and $\bar{u}=(\bar{m}, \bar{w}, \bar{q}, \bar{p})$ both belong to $\mathbb{E}$ with norm less than $R$. We use the notation introduced in (1.10) to define

$$
A_{\delta}[u] v:=-\nabla_{x} \cdot\left(\delta(\zeta(m)) \nabla_{x} v\right)+\chi(\zeta(m)) \nabla_{x} \zeta(m) \cdot \nabla_{x} v, \quad v \in W_{\varrho, \mathscr{B}}^{2}
$$

and notice that

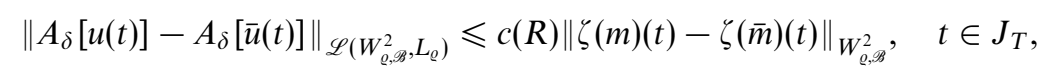

since $\delta_{j}, \delta_{j}^{\prime}$, and $\chi_{j}$ are uniformly Lipschitz continuous on compact subset of $\mathbb{R}^{+}$due to assumptions $\left(A_{1}\right)$ and $\left(A_{3}\right)$. Furthermore, since $\delta(\zeta(m)) \in C^{1}\left(\bar{\Omega}, \mathbb{R}^{N}\right)$ and $\delta_{j}(\zeta(m))$ is uniformly bounded away from zero on $J_{T} \times \bar{\Omega}$ for $j=1, \ldots, N$ by assumption $\left(A_{1}\right)$, it follows from [1, Thm.2.4] and (3.2) that

$$
\left[t \mapsto A_{\delta}[u(t)]\right] \in C^{1-\bar{\mu}}\left(J_{T}, \mathscr{H}^{+}\left(W_{\varrho, \mathscr{B}}^{2}, L_{\varrho}\right)\right) \text { with }\left\|A_{\delta}[u]\right\|_{C^{1-\bar{\mu}}\left(J_{T}, \mathscr{H}^{+}\left(W_{\varrho, g}^{2}, L_{\varrho}\right)\right)} \leqslant c(T, R) .
$$

Defining

$$
P(t, x):=\int_{0}^{\infty} p(t, a, x) \mathrm{d} a, \quad Q(t, x):=\int_{0}^{\infty} q(t, a, x) \mathrm{d} a
$$

and $\mathfrak{b}[u](t):=[a \mapsto \mathfrak{b}(a, w(t, \cdot), P(t, \cdot), Q(t, \cdot))]$, Lemma 2.7 and assumption $\left(A_{7}\right)$ imply that

$$
\mathfrak{b} \in C_{b}^{1-}\left(\mathbb{E}, F_{\bar{\eta}}\right) \quad \text { with } \quad\|\mathfrak{b}[u]\|_{F_{\bar{\eta}}} \leqslant c(R) .
$$

Hence $A_{\delta}[u]$ and $\mathfrak{b}[u]$ satisfy (2.4) and (2.5). By $\left\{\mathscr{U}_{[u]}(t, s) ;(t, s) \in \Delta_{T}\right\}$ we then denote the evolution operator induced by $A_{\delta}[u]$ and $\mathfrak{b}[u]$ (cf. Proposition 2.1), i.e., we put $\mathscr{U}_{[u]}:=\mathscr{U}_{\left[A_{\delta}[u], \mathfrak{b}[u]\right]}$. Setting

$$
A_{\gamma}[u] v:=-\nabla_{x} \cdot\left(\gamma(\zeta(m)) \nabla_{x} v\right), \quad v \in \mathbb{W}_{\varrho, \mathscr{B}}^{2},
$$


and noticing that the differential operator merely acts on the spatial variable $x$, it is easily seen that from (3.2)

$$
\left[t \mapsto A_{\gamma}[u(t)]\right] \in C^{1-\bar{\mu}}\left(J_{T}, \mathscr{H}^{+}\left(\mathbb{W}_{\varrho, \mathscr{B}}^{2}, \mathbb{L}_{\varrho}\right)\right)
$$

with

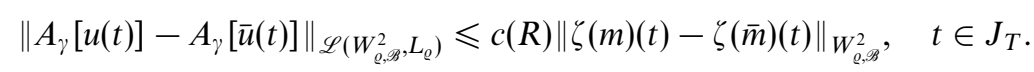

Hence $-A_{\gamma}[u]$ generates an evolution system $\left\{U_{A_{\gamma}[u]}(t, s) ;(t, s) \in \Delta_{T}\right\}$ on $\mathbb{L}_{\varrho}$ according to [3]. Invoking [3, II.Lem.5.1.4] and Lemma 2.6 we deduce that

$$
\begin{aligned}
\| U_{A_{\gamma}[u]}(t, s)-U_{A_{\gamma}[\bar{u}]} & (t, s) \|_{\mathscr{L}\left(\mathbb{W}_{\varrho, \mathscr{B}}^{2 \sigma}, \mathbb{W}_{\varrho, \mathscr{B}}^{2 \tau}\right)} \\
& \leqslant c(R)(t-s)^{\sigma-\tau} \max _{s \leqslant r \leqslant t}\left\|A_{\gamma}[u(r)]-A_{\gamma}[\bar{u}(r)]\right\|_{\mathscr{L}\left(\mathbb{W}_{Q, \mathscr{B}}^{2}, \mathbb{L}_{\varrho}\right)} \\
& \leqslant c(T, R)(t-s)^{\sigma-\tau}\|u-\bar{u}\|_{\mathbb{E}}
\end{aligned}
$$

for $(t, s) \in \Delta_{T}^{*}$, and $0<\sigma \leqslant 1,0 \leqslant \tau<1$ with $2 \sigma, \tau \neq 1+1 / \varrho$. Moreover, [3, II.Lem.5.1.3] warrants that $U_{A_{\gamma}[u]}$ satisfies the analogue estimates as in (A 5) for the $\mathbb{W}_{\varrho, \mathscr{B}}^{2 \sigma}$-spaces. Clearly,

$$
-A_{\tau}:=\left[v \mapsto \nabla_{x} \cdot\left(\tau(\cdot) \nabla_{x} v\right)\right] \in \mathscr{H}^{+}\left(W_{\varrho, \mathscr{B}}^{2}, L_{\varrho}\right)
$$

generates a positive analytic semigroup $\left\{U_{A_{\tau}}(t) ; t \in J_{T}\right\}$ on $L_{\varrho}$ for $\tau=\alpha, \beta$. We then put

$$
\begin{aligned}
& R_{1}(u):=\Theta(P, Q)-h m, \\
& R_{2}(u):=\Gamma(\cdot, \zeta(m))-\Lambda(P, Q) w-e w, \\
& R_{3}(u):=\Sigma(\cdot, w, P, Q) p-(\Phi+\Upsilon)(\cdot, w, P, Q) q, \\
& R_{4}(u):=-p \nabla_{x} \cdot\left(\chi(\zeta(m)) \nabla_{x} \zeta(m)\right)-(b+\Sigma+\Psi)(\cdot, w, P, Q) p+\Phi(\cdot, w, P, Q) q
\end{aligned}
$$

again using the notation as in (1.9)-(1.12). Furthermore, for operators $V=V(t), U=$ $U(t, s)$, and a function $u=u(s)$ we put

$$
V \star u(t):=\int_{0}^{t} V(t-s) u(s) \mathrm{d} s, \quad U \star u(t):=\int_{0}^{t} U(t, s) u(s) \mathrm{d} s
$$

whenever these integrals make sense. Given

$$
u^{0}:=\left(f^{0}, m^{0}, w^{0}, q^{0}, p^{0}\right) \in X=W_{\varrho, \mathscr{B}}^{2+\epsilon} \times W_{\varrho, \mathscr{B}}^{2 \lambda} \times W_{\varrho, \mathscr{B}}^{2 \xi} \times \mathbb{W}_{\varrho, \mathscr{B}}^{2 \xi} \times \mathbb{W}_{\varrho, \mathscr{B}}^{2 \xi}
$$

with $\left\|u^{0}\right\|_{X} \leqslant R_{0}$, we define $F(u):=\left(F_{1}(u), \ldots, F_{4}(u)\right)$ by

$$
\begin{aligned}
& F_{1}(u)(t):=U_{A_{\alpha}}(t) m^{0}+U_{A_{\alpha}} \star R_{1}(u)(t), \\
& F_{2}(u)(t):=U_{A_{\beta}}(t) w^{0}+U_{A_{\beta}} \star R_{2}(u)(t), \\
& F_{3}(u)(t):=U_{A_{\gamma}[u]}(t, 0) q^{0}+U_{A_{\gamma}[u]} \star R_{3}(u)(t), \\
& F_{4}(u)(t):=\mathscr{U}_{[u]}(t, 0) p^{0}+\mathscr{U}_{[u]} \star R_{4}(u)(t),
\end{aligned}
$$

for $u \in \mathbb{E}$ and $t \in J_{T}$. Note that $U_{A_{\alpha}}(\cdot) m^{0} \in E_{1}$ by Lemma 2.5. Also observe that (3.1) and $\left[2\right.$, Thm.4.1] ensure that pointwise multiplication $C^{\epsilon}(\bar{\Omega}) \times W_{\varrho}^{2+2 v} \rightarrow W_{\varrho}^{2 \bar{v}}$ is continuous. 
Hence, due to assumption $\left(A_{2}\right)$, it follows from Lemma 2.7 that

$$
R_{1} \in C_{b}^{1-}\left(\mathbb{E}, C_{\mu}\left(\dot{J}_{T}, W_{\varrho, \mathscr{B}}^{2 \bar{v}}\right)\right)
$$

from which we conclude that

$$
\left\|F_{1}(u)-F_{1}(\bar{u})\right\|_{E_{1}}=\left\|U_{A_{\alpha}} \star\left(R_{1}(u)-R_{1}(\bar{u})\right)\right\|_{E_{1}} \leqslant c(R) T^{v-\bar{v}}\|u-\bar{u}\|_{\mathbb{E}}
$$

and

$$
\left\|F_{1}(u)\right\|_{E_{1}} \leqslant c(T)\left\|m^{0}\right\|_{W_{\varrho, B}^{2 \eta}}+c(R) T^{v-\bar{v}}
$$

for $\|u\|_{\mathbb{E}},\|\bar{u}\|_{\mathbb{E}} \leqslant R$ owing to (A 5). Next, $w^{0} \in W_{\varrho, \mathscr{B}}^{2 \xi}$ entails $U_{A_{\beta}}(\cdot) w^{0} \in E_{2}$. Assumptions $\left(A_{2}\right),\left(A_{5}\right),\left(A_{6}\right)$, and Lemma 2.6 imply that $R_{2} \in C_{b}^{1-}\left(\mathbb{E}, C\left(J_{T}, L_{\varrho}\right)\right)$, whence

$$
\left\|F_{2}(u)-F_{2}(\bar{u})\right\|_{E_{2}}=\left\|U_{A_{\beta}} \star\left(R_{2}(u)-R_{2}(\bar{u})\right)\right\|_{E_{2}} \leqslant c(T, R) T^{1-\eta}\|u-\bar{u}\|_{\mathbb{E}}
$$

for $\|u\|_{\mathbb{E}},\|\bar{u}\|_{\mathbb{E}} \leqslant R$ by (A 5). Moreover,

$$
\left.\| F_{2}(u)\right)\left\|_{E_{2}} \leqslant c(T)\right\| w^{0} \|_{W_{\varrho, g}^{2 \eta}}+c(T, R) T^{1-\eta}
$$

From (3.6) we obtain

$$
\left[t \mapsto U_{A_{\gamma}[u]}(t, 0) q^{0}\right] \in C\left(J_{T}, \mathbb{W}_{\varrho, \mathscr{B}}^{2 \xi}\right) \hookrightarrow E_{3}
$$

due to $q^{0} \in \mathbb{W}_{\varrho, \mathscr{B}}^{2 \xi}$. Analogously to (3.5) and by using (1.12) we may interpret $b, \Sigma, \Psi, \Phi$, and $Y$ as members of $C_{b}^{1-}\left(\mathbb{E}, F_{\bar{\eta}}\right)$ and so, owing to (A 4$)$ (with $\bar{\eta}$ instead of $\eta$ ),

$$
R_{3} \in C_{b}^{1-}\left(\mathbb{E}, C\left(J_{T}, \mathbb{W}_{\varrho, \mathscr{B}}^{2 \bar{\eta}}\right)\right)
$$

Therefore, invoking (3.7), the analogue of (A 5) for $U_{A_{\gamma}[u]}$, and recalling that $\left\|q^{0}\right\|_{\mathbb{W}_{\varrho, \mathscr{B}}^{2 \xi}} \leqslant R_{0}$ and $\|u\|_{\mathbb{E}},\|\bar{u}\|_{\mathbb{E}} \leqslant R$, we deduce that, for $t \in J_{T}$ and $\varepsilon>0$ small,

$$
\begin{aligned}
\| F_{3}(u)(t)- & F_{3}(\bar{u})(t) \|_{\mathbb{W}_{\varrho, \mathscr{B}}^{2 \eta}} \\
\leqslant & \left\|U_{A_{\gamma}[u]}(t, 0)-U_{A_{\gamma}[\bar{u}]}(t, 0)\right\|_{\mathscr{L}\left(\mathbb{W}_{\varrho, \mathscr{B}}^{2 \xi}, \mathbb{W}_{\varrho, \mathscr{B}}^{2 \eta}\right)}\left\|q^{0}\right\|_{\mathbb{W}_{\varrho, \mathscr{B}}^{2 \xi}} \\
& +\int_{0}^{t}\left\|U_{A_{\gamma}[u]}(t, s)-U_{A_{\gamma}[\bar{u}]}(t, s)\right\|_{\mathscr{L}\left(\mathbb{W}_{\varrho, \mathscr{B}}^{2 \bar{\eta}}, \mathbb{W}_{\varrho, \mathscr{B}}^{2 \eta}\right)}\left\|R_{3}(u(s))\right\|_{\mathbb{W}_{\varrho, \mathscr{B}}^{2 \bar{\eta}}} \mathrm{d} s \\
& +\int_{0}^{t}\left\|U_{A_{\gamma}[\bar{u}]}(t, s)\right\|_{\mathscr{L}\left(\mathbb{W}_{\varrho, \mathscr{P}}^{2 \bar{\eta}}, \mathbb{W}_{\varrho, \mathscr{B}}^{2 \eta}\right)}\left\|R_{3}(u(s))-R_{3}(\bar{u}(s))\right\|_{\mathbb{W}_{\varrho, \mathscr{P}}^{2 \bar{\eta}}} \mathrm{d} s \\
\leqslant & c\left(T, R, R_{0}\right) t^{\xi-\eta}\|u-\bar{u}\|_{\mathbb{E}}+c(T, R) \int_{0}^{t}(t-s)^{\bar{\eta}-\eta-\varepsilon} \mathrm{d} s\|u-\bar{u}\|_{\mathbb{E}} \\
\leqslant & c\left(T, R, R_{0}\right) t^{\xi-\eta}\|u-\bar{u}\|_{\mathbb{E} .}
\end{aligned}
$$


Next observe that $\left\|U_{A_{\gamma}[0]}(t, 0)\right\|_{\mathscr{L}\left(\mathbb{W}_{\varrho, \mathscr{B}}^{2 \eta}\right)} \leqslant c\left(T, R_{0}\right)$ due to [3, II.Lem.5.1.3] and $\zeta(0)=f^{0}$. Thus, since $F_{3}(0)(t)=U_{A_{\gamma}[0]}(t, 0) q^{0}$,

$$
\begin{aligned}
\left\|F_{3}(u)(t)\right\|_{\mathbb{W}_{\varrho, \mathscr{B}}^{2 \eta}} & \leqslant\left\|F_{3}(u)(t)-F_{3}(0)(t)\right\|_{\mathbb{W}_{\varrho, 9 B}^{2 \eta}}+\left\|U_{A_{\gamma}[0]}(t, 0) q^{0}\right\|_{\mathbb{W}_{\varrho, g B}^{2 \eta}} \\
& \leqslant c\left(T, R, R_{0}\right) T^{\xi-\eta}+c\left(T, R_{0}\right)
\end{aligned}
$$

for $t \in J_{T}$. Due to $p^{0} \in \mathbb{W}_{\varrho, \mathscr{B}}^{2 \xi}$ it follows from Proposition 2.1 that

$$
\left[t \mapsto \mathscr{U}_{[u]}(t, 0) p^{0}\right] \in C\left(J_{T}, \mathbb{W}_{\varrho, \mathscr{B}}^{2 \xi}\right)
$$

Let $0<r<\bar{r}<v$ and note that pointwise multiplications $W_{\varrho}^{1+2 \bar{r}} \times W_{\varrho}^{1+2 \bar{r}} \hookrightarrow W_{\varrho}^{1+2 \bar{r}}$ and $W_{\varrho}^{2 \eta} \times W_{\varrho}^{2 \bar{r}} \hookrightarrow W_{\varrho}^{2 r}$ are continuous by [2, Thm.4.1] since $\varrho>n$ and $2 \eta>n / \varrho$. Hence, according to Lemma 2.7 and assumption $\left(A_{3}\right)$ we have

$$
\left[u \mapsto \nabla_{x} \cdot\left(\chi(\zeta(m)) \nabla_{x} \zeta(m)\right) p\right] \in C_{b}^{1-}\left(\mathbb{E}, C\left(J_{T}, \mathbb{W}_{\varrho, \mathscr{B}}^{2 r}\right)\right)
$$

and thus, taking into account $b, \Sigma, \Psi, \Phi \in C_{b}^{1-}\left(\mathbb{E}, F_{\bar{\eta}}\right)$ and (A 4) in the Appendix (with $\eta$ replaced by $\bar{\eta}$ )

$$
R_{4} \in C_{b}^{1-}\left(\mathbb{E}, C\left(J_{T}, \mathbb{W}_{\varrho, \mathscr{B}}^{2 r}\right)\right)
$$

Next, we use (2.6) and (2.7) of Proposition 2.1 and (3.2), (3.3), (3.5), and (3.18). We then compute for $\left\|p^{0}\right\|_{\mathbb{W}_{\varrho, \mathscr{B}}^{2 \xi}} \leqslant R_{0},\|u\|_{\mathbb{E}},\|\bar{u}\|_{\mathbb{E}} \leqslant R$, and $t \in J_{T}$ that (with $\varepsilon>0$ small)

$$
\begin{aligned}
& \left\|F_{4}(u)(t)-F_{4}(\bar{u})(t)\right\|_{\mathbb{W}_{Q, \mathscr{B}}^{2 \eta}} \\
& \leqslant\left\|\mathscr{U}_{[u]}(t, 0)-\mathscr{U}_{[\bar{u}]}(t, 0)\right\|_{\mathscr{L}\left(\mathbb{W}_{\varrho, \mathscr{B}}^{2 \xi}, \mathbb{W}_{\varrho, \mathscr{B}}^{2 \eta}\right)}\left\|p^{0}\right\|_{\mathbb{W}_{\varrho, \mathscr{B}}^{2 \xi}} \\
& +\int_{0}^{t}\left\|\mathscr{U}_{[u]}(t, s)-\mathscr{U}_{[\bar{u}]}(t, s)\right\|_{\mathscr{L}\left(\mathbb{W}_{\varrho, \mathscr{B}}^{2 r}, \mathbb{W}_{\varrho, \mathscr{B}}^{2 \eta}\right)}\left\|R_{4}(u(s))\right\|_{\mathbb{W}_{\varrho, \mathscr{B}}^{2 r}} \mathrm{~d} s \\
& +\int_{0}^{t}\left\|\mathscr{U}_{[\bar{u}]}(t, s)\right\|_{\mathscr{L}\left(\mathbb{W}_{\varrho, \mathscr{B}}^{2 r}, \mathbb{W}_{\varrho, \mathscr{B}}^{2 \eta}\right)}\left\|R_{4}(u(s))-R_{4}(\bar{u}(s))\right\|_{\mathbb{W}_{\varrho, \mathscr{B}}^{2 r}} \mathrm{~d} s \\
& \leqslant c\left(T, R, R_{0}\right) t^{\xi-\eta}\|u-\bar{u}\|_{\mathbb{E}}+c(T, R) \int_{0}^{t}(t-s)^{r-\eta-\varepsilon} \mathrm{d} s\|u-\bar{u}\|_{\mathbb{E}} \\
& \leqslant c\left(T, R, R_{0}\right) t^{\xi-\eta}\|u-\bar{u}\|_{\mathbb{E}} .
\end{aligned}
$$

To find a bound on $F_{4}(u)$ we note that $\mathfrak{b}[0] \in F_{\eta}$ and, since $\zeta(0)=f^{0}$ and $\left\|f^{0}\right\|_{W_{Q, \mathscr{B}}^{2}} \leqslant R_{0}$, that $\left\|A_{\delta}[0]\right\|_{\mathscr{L}\left(W_{Q, \mathscr{B}}^{2}, L_{\varrho}\right)} \leqslant c\left(R_{0}\right)$. Therefore, $\left\|\mathscr{U}_{[0]}(t, 0)\right\|_{\mathscr{L}\left(\mathbb{W}_{Q, \mathscr{B}}^{2 \eta}\right)} \leqslant c\left(T, R_{0}\right)$ for $t \in J_{T}$ by (2.6) from which

$$
\begin{aligned}
\left\|F_{4}(u)(t)\right\|_{\mathbb{W}_{\varrho, \mathscr{B}}^{2 \eta}} & \leqslant\left\|F_{4}(u)(t)-F_{4}(0)(t)\right\|_{\mathbb{W}_{\varrho, \mathscr{B}}^{2 \eta}}+\left\|\mathscr{U}_{[0]}(t, 0)\right\|_{\mathscr{L}\left(\mathbb{W}_{\varrho, \mathscr{B}}^{2 \eta}\right)}\left\|p^{0}\right\|_{\mathbb{W}_{\varrho, \mathscr{B}}^{2 \eta}} \\
& \leqslant c\left(T, R, R_{0}\right) T^{\xi-\eta}+c\left(T, R_{0}\right)
\end{aligned}
$$


for $t \in J_{T}$. Gathering together (3.10)-(3.13), (3.16), (3.17), (3.19), and (3.20) we deduce that $F: \mathbb{E} \rightarrow \mathbb{E}$ satisfies

$$
\begin{aligned}
\|F(u)-F(\bar{u})\|_{\mathbb{E}} & \leqslant c\left(T, R_{0}, R\right) T^{\vartheta}\|u-\bar{u}\|_{\mathbb{E}}, \\
\|F(u)\|_{\mathbb{E}} & \leqslant c_{0}\left(T, R_{0}\right)+c\left(T, R_{0}, R\right) T^{\vartheta},
\end{aligned}
$$

provided that $\|u\|_{\mathbb{E}},\|\bar{u}\|_{\mathbb{E}} \leqslant R$ and $\left\|u^{0}\right\|_{X} \leqslant R_{0}$, where $\vartheta:=(\bar{v}-v) \wedge(\xi-\eta)>0$. Note that the constant $c_{0}\left(T, R_{0}\right)$ in (3.22) does not depend on $R$. We recall that $T, R, R_{0}>0$ were arbitrary. Hence, given any $R_{0}>0$ we may define $R:=c_{0}\left(1, R_{0}\right)+1$ and choose $T:=T\left(R_{0}\right) \in(0,1]$ small to obtain that $F$ is a contraction from the ball in $\mathbb{E}=\mathbb{E}_{T}$ centred at 0 of radius $R_{0}$ into itself. This yields a unique fixed point $u=(m, w, q, p)$ of $F$ in this ball. Obviously, $(\zeta(m), m, w, q, p)$ is then the unique mild solution to (1.14)-(1.21) on $[0, T]$. This solution can be extended to a maximal solution on some maximal interval of existence $J$ by uniqueness. Since $T=T\left(R_{0}\right)$ was chosen only depending on $R_{0}$ with $\left\|u^{0}\right\|_{X} \leqslant R_{0}$, we may conclude that $J=\mathbb{R}^{+}$provided we can show that

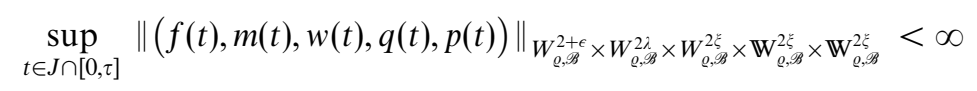

for each $\tau>0$, where $f:=\zeta(m)$.

\subsection{Proof of theorem 3.1: Regularity}

Improving the regularity of the solution found in the previous subsection is now standard. First note that as in [30, Lem.2.2(i)] we have $f \in C^{1}\left(\dot{J}, W_{\varrho, \mathscr{B}}^{2}\right) \cap C\left(J, W_{\varrho, \mathscr{B}}^{2}\right)$. Writing $R_{j}(t):=R_{j}(u(t)), j=1, \ldots, 4$, we infer from (3.9) that $R_{1} \in C\left(\dot{J}, W_{\varrho, \mathscr{B}}^{2 \bar{v}}\right)$ with $\bar{v}>0$ and thus, from [3, II.Thm.1.2.2],

$$
m \in C^{1}\left(\dot{J}, L_{\varrho}\right) \cap C\left(\dot{J}, W_{\varrho, \mathscr{B}}^{2}\right) \cap C\left(J, W_{\varrho, \mathscr{B}}^{2 \lambda}\right)
$$

is a classical solution of (1.15). Next, integrating the mild formulation of (1.17) with respect to $a>0$ we obtain

$$
Q(t):=\int_{0}^{\infty} q(t, a) \mathrm{d} a=U_{A_{\gamma}[u]}(t, 0) \int_{0}^{\infty} q^{0}(a) \mathrm{d} a+\int_{0}^{t} U_{A_{\gamma}[u]}(t, s) \int_{0}^{\infty} R_{3}(u(s))(a) \mathrm{d} a \mathrm{~d} s,
$$

for $t \in J$, where $U_{A_{\gamma}[u]}$ has to be interpreted here as the evolution operator on $L_{\varrho}$ corresponding to $A_{\gamma}[u] \in C^{1-\bar{\mu}}\left(J, \mathscr{H}\left(W_{\varrho, \mathscr{B}}^{2}, L_{\varrho}\right)\right)$. Owing to

$$
\int_{0}^{\infty} q^{0} \mathrm{~d} a \in W_{\varrho, \mathscr{B}}^{2 \xi} \quad \text { and } \quad \int_{0}^{\infty} R_{3}(u)(a) \mathrm{d} a \in C\left(J, L_{\varrho}\right)
$$

it follows from [3, II.Thm.5.3.1] that

$$
Q \in C^{\xi-\eta}\left(J, W_{\varrho, \mathscr{B}}^{2 \eta}\right)
$$


where still $2 \eta \in(n / \varrho, 2 \xi) \backslash\{1+1 / \varrho\}$. Similarly, invoking Lemma 2.3 we deduce that

$$
P:=\int_{0}^{\infty} p \mathrm{~d} a \in C\left(J, L_{\varrho}\right)
$$

is the mild solution to the Cauchy problem

$$
\dot{P}+A_{\delta}[u(t)] P=H(t), \quad t \in \dot{J}, \quad P(0)=\int_{0}^{\infty} p^{0} \mathrm{~d} a,
$$

where

$$
H(t):=\int_{0}^{\infty} R_{4}(u(s))(a) \mathrm{d} a+2 \int_{0}^{\infty} \mathfrak{b}[u(t)](a) p(t, a) \mathrm{d} a, \quad t \in J .
$$

Since $H \in C\left(J, W_{\varrho, \mathscr{B}}^{2 r}\right)$ due to (3.18), (3.5), and (A4) (from the Appendix) and since $P(0) \in W_{\varrho, \mathscr{B}}^{2 \xi}$ we conclude again from [3, II.Thm.5.3.1] that

$$
P \in C^{\xi-\eta}\left(J, W_{\varrho, \mathscr{B}}^{2 \eta}\right) \cap C^{1}\left(\dot{J}, L_{\varrho}\right) \cap C\left(\dot{J}, W_{\varrho, \mathscr{B}}^{2}\right) \cap C\left(J, W_{\varrho, \mathscr{B}}^{2 \xi}\right)
$$

is the classical solution of (3.25). Hence, Lemma 2.7 together with (3.24) and (3.26) yield that $\Lambda(P, Q)$ belongs to $C^{\xi-\eta}\left(J, W_{\varrho, \mathscr{B}}^{2 \bar{\eta}}\right)$ with $\bar{\eta} \in(0, \eta)$. We observe that $w^{0} \in W_{\varrho, \mathscr{B}}^{2 \xi}$ and $R_{2} \in C\left(J, L_{\varrho}\right)$ entail $w \in C^{\xi-\eta}\left(J, W_{\varrho, \mathscr{B}}^{2 \eta}\right)$ and so we derive from Lemma 2.6 and $\left(A_{5}\right)$ that $R_{2} \in C^{\rho}\left(J, L_{\varrho}\right)$ for some $\rho>0$. Therefore,

$$
w \in C^{1}\left(\dot{J}, L_{\varrho}\right) \cap C\left(\dot{J}, W_{\varrho, \mathscr{B}}^{2}\right) \cap C\left(J, W_{\varrho, \mathscr{B}}^{2 \xi}\right)
$$

is the classical solution of (1.16). From (3.15) it follows $R_{3} \in C\left(J, \mathbb{W}_{\varrho, \mathscr{B}}^{2 \bar{\eta}}\right)$ and hence, due to (3.6) and $q^{0} \in \mathbb{W}_{\varrho, \mathscr{B}}^{2 \xi}$, we obtain that

$$
q \in C^{1}\left(\dot{J}, \mathbb{L}_{\varrho}\right) \cap C\left(\dot{J}, \mathbb{W}_{\varrho, \mathscr{B}}^{2}\right) \cap C\left(J, \mathbb{W}_{\varrho, \mathscr{B}}^{2 \xi}\right)
$$

solves (1.17) classically. It remains to observe that $p \in C\left(J, \mathbb{W}_{\varrho, \mathscr{B}}^{2 \xi}\right)$ is due to the fact that $p^{0} \in \mathbb{W}_{\varrho, \mathscr{B}}^{2 \xi}$ and that $\mathscr{U}_{[u]}$ is strongly continuous on $\mathbb{W}_{\varrho, \mathscr{B}}^{2 \xi}$ by Proposition 2.1 .

\subsection{Proof of theorem 3.1: Positivity}

For nonnegative initial values $\left(f^{0}, m^{0}, w^{0}, q^{0}, p^{0}\right)$ as in (3.8) we denote by $(f, u)=$ $(f, m, w, q, p)$ the maximal solution to (1.14)-(1.21) obtained in Subsection 3.1. We fix $T \in \dot{J}$ and set $J_{T}:=[0, T]$. Then $w, Q, P \in C\left(J_{T}, C(\bar{\Omega})\right)$ since $W_{\varrho, \mathscr{B}}^{2 \xi} \hookrightarrow C(\bar{\Omega})$ and thus $\Xi \in C\left(J_{T} \times \mathbb{R}^{+} \times \bar{\Omega}\right)$, where

$$
\Xi(t, a, x):=\Xi(a, w(t, x), P(t, x), Q(t, x)), \quad(t, a, x) \in J_{T} \times \mathbb{R}^{+} \times \bar{\Omega},
$$

for $\Xi \in\{b, \Sigma, \Phi, \Psi, \Upsilon\}$. We put $z:=\nabla_{x} \cdot\left(\chi(f) \nabla_{x} f\right)$ and obtain $z \in C\left(J_{T}, L_{\varrho}\right)$ from (3.18). Let then $z_{j} \in C\left(J_{T}, C(\bar{\Omega})\right)$ be such that $z_{j} \rightarrow z$ in $C\left(J_{T}, L_{\varrho}\right)$ as $j \rightarrow \infty$. We set

$$
M_{1}:=-\Upsilon-\Phi, \quad M_{2}:=\Sigma, \quad M_{3}:=\Phi, \quad M_{4}:=-z_{j}-b-\Sigma-\Psi .
$$


Due to Lemma 2.4 there exists for each $j \in \mathbb{N}$ a unique solution $\left(q_{j}, p_{j}\right) \in C\left(J_{T}, \mathbb{L}_{\varrho}^{+} \times \mathbb{L}_{\varrho}^{+}\right)$ of

$$
\begin{aligned}
& q_{j}(t)=U_{A_{y}[u]}(t, 0) q^{0}+U_{A_{\gamma}[u]} \star\left(M_{1} q_{j}+M_{2} p_{j}\right)(t), \quad t \in J_{T}, \\
& p_{j}(t)=\mathscr{U}_{[u]}(t, 0) p^{0}+\mathscr{U}_{[u]} \star\left(M_{3} q_{j}+M_{4} p_{j}\right)(t), \quad t \in J_{T} .
\end{aligned}
$$

It is immediate from Proposition 2.1(iv) and (2.6) that $p_{j} \in C\left(J_{T}, \mathbb{W}_{\varrho, \mathscr{B}}^{2 \xi}\right)$. Using the corresponding mild formulation of (1.17) and (1.18) it easily follows that $q_{j} \rightarrow q$ in $C\left(J_{T}, \mathbb{L}_{\varrho}\right)$ and $p_{j} \rightarrow p$ in $C\left(J_{T}, \mathbb{W}_{\varrho, \mathscr{B}}^{2 \xi}\right)$ since $z_{j} \rightarrow z$. Therefore, $q(t), p(t) \in \mathbb{L}_{\varrho}^{+}$for $t \in J_{T}$, and hence for all $t \in J$ since $T \in \dot{J}$ was arbitrary. That $f \geqslant 0, m \geqslant 0$, and $w \geqslant 0$ is obvious.

\subsection{Proof of theorem 3.1: Global existence}

Suppose now that $\left(A_{1}\right)-\left(A_{10}\right)$ hold. We again denote by $(f, u)=(f, m, w, q, p)$ the maximal solution to (1.14)-(1.21) on the interval $J$ obtained in Section 3.1 corresponding to nonnegative initial values $\left(f^{0}, m^{0}, w^{0}, q^{0}, p^{0}\right)$ as in (3.8). In order to prove (3.22) we proceed similarly as in [30] and [29]. Let $\tau>0$ be arbitrary and put $J_{\tau}:=J \cap[0, \tau]$. First observe that

$$
\|f(t)\|_{\infty} \leqslant\left\|f^{0}\right\|_{\infty}, \quad t \in J
$$

and so, due to (1.16),

$$
\|w(t)\|_{\infty} \leqslant c(\tau), \quad t \in J_{\tau} .
$$

Invoking $\left(A_{9}\right)$ we thus infer from (3.23) and (3.25) that

$$
\begin{aligned}
\frac{\mathrm{d}}{\mathrm{d} t} \sum_{j=1}^{N} \int_{\Omega}\left(P_{j}(t)+Q_{j}(t)\right) \mathrm{d} x & \leqslant 2 \sum_{j, l=1}^{N} \int_{\Omega} \int_{0}^{\infty} b_{l}(a, w, P, Q) \psi_{l, j}(a, w, P, Q) p_{l}(t, a, x) \mathrm{d} a \mathrm{~d} x \\
& \leqslant c(\tau) \sum_{j=1}^{N} \int_{\Omega} P_{j}(t) \mathrm{d} x
\end{aligned}
$$

for $t \in J_{\tau}$. Therefore

$$
\|P(t)\|_{L_{1}}+\|Q(t)\|_{L_{1}} \leqslant c(\tau), \quad t \in J_{\tau}
$$

We fix $j \in\{1, \ldots, N\}$. Owing to (3.27) and $\left(A_{1}\right)$ there hold

$$
\delta_{j}(f(t)) \geqslant \underline{\delta}>0, \quad\left\|\frac{\chi_{j}(f(t))}{\delta_{j}(f(t))}\right\|_{\infty} \leqslant c_{0}, \quad t \in J .
$$

We then introduce the function $\phi_{j}(z):=\exp \left(\int_{0}^{z} \frac{\chi_{j}(r)}{\delta_{j}(r)} \mathrm{d} r\right), z \geqslant 0$, and observe that $\phi_{j} \in C^{1}$ solves

$$
\phi_{j}^{\prime}=\frac{\chi_{j}}{\delta_{j}} \phi_{j}, \quad \phi_{j}(0)=1
$$


and satisfies

$$
1 \leqslant \phi_{j}(f(t)) \leqslant e^{c_{0}\left\|f^{0}\right\|_{\infty}}, \quad t \in J
$$

To simplify the notation in the sequel we omit the arguments $w, P, Q$ and merely write $\Sigma_{j}$ etc. instead of $\Sigma_{j}(a, w, P, Q)$. From (3.25) and (3.31) it follows that

$$
\begin{aligned}
\partial_{t} \frac{P_{j}}{\phi_{j}(f)}= & \frac{1}{\phi_{j}(f)} \nabla_{x} \cdot\left(\delta_{j}(f) \chi(f) \nabla_{x} \frac{P_{j}}{\phi_{j}(f)}\right)+\frac{\chi_{j}(f)}{\delta_{j}(f)} \frac{P_{j}}{\phi_{j}(f)} k m f+\frac{1}{\phi_{j}(f)} \int_{0}^{\infty} \Phi_{j} q_{j} \mathrm{~d} a \\
& -\frac{1}{\phi_{j}(f)} \int_{0}^{\infty}\left(\Sigma_{j}+\Psi_{j}+b_{j}\right) p_{j} \mathrm{~d} a+\frac{2}{\phi_{j}(f)} \sum_{l=1}^{N} \int_{0}^{\infty} b_{l} \psi_{l, j} p_{l} \mathrm{~d} a .
\end{aligned}
$$

We next prove the following auxiliary result which is in the spirit of [30, Prop.5.1] and [29, Lem.3.3].

Lemma 3.3 If $\|P(t)\|_{L_{\rho}} \leqslant c(\tau), t \in J_{\tau}$, for some $\rho \in[1, \varrho)$, then $\|P(t)\|_{L_{\sigma}} \leqslant c(\tau), t \in J_{\tau}$, provided that $\sigma \in(\rho, 2 \rho \wedge \varrho]$ is such that $\frac{n \sigma}{n \sigma+2 \rho}<1+\frac{2}{n}-\frac{1}{\rho}$.

Proof Let $\|P(t)\|_{L_{\rho}} \leqslant c(\tau), t \in J_{\tau}$, for some $\rho \in[1, \varrho)$ and let $\sigma \in(\rho, 2 \rho \wedge \varrho]$ and $r>1$ be such that

$$
\frac{n \sigma}{n \sigma+2 \rho}<\frac{1}{r}<1+\frac{2}{n}-\frac{1}{\rho} .
$$

Fix $j \in\{1, \ldots, N\}$. We use $\left(A_{9}\right),(3.27),(3.28),(3.30)$, and (3.33) to deduce as in [30, Prop.5.1], [29, Lem.3.3] that

$$
\begin{aligned}
\frac{\mathrm{d}}{\mathrm{d} t} \int_{\Omega} \phi_{j}(f)\left(\frac{P_{j}}{\phi_{j}(f)}\right)^{\sigma} \mathrm{d} x \leqslant & -\sigma(\sigma-1) \underline{\delta} \int_{\Omega} \phi_{j}(f)\left|\nabla_{x}\left(\frac{P_{j}}{\phi_{j}(f)}\right)^{\sigma / 2}\right|^{2} \mathrm{~d} x \\
& +c(\tau) \int_{\Omega} m\left(\frac{P_{j}}{\phi_{j}(f)}\right)^{\sigma} \mathrm{d} x+c(\tau) \int_{\Omega} Q_{j}\left(\frac{P_{j}}{\phi_{j}(f)}\right)^{\sigma-1} \mathrm{~d} x \\
& +c(\tau) \sum_{l=1}^{N} \int_{\Omega} P_{l}\left(\frac{P_{j}}{\phi_{j}(f)}\right)^{\sigma-1} \mathrm{~d} x
\end{aligned}
$$

for $t \in \dot{J}_{\tau}$. Next observe that from $\left(A_{9}\right)$

$$
\begin{aligned}
\partial_{t} Q_{j}-\nabla_{x} \cdot\left(\gamma_{j}(f) \nabla_{x} Q_{j}\right) & \leqslant \int_{0}^{\infty} \Sigma_{j}(a, w, P, Q) p_{j} \mathrm{~d} a \leqslant c(\tau) P_{j}, \\
\partial_{t} m-\nabla_{x} \cdot\left(\alpha \nabla_{x} m\right) & \leqslant \Theta(P, Q) .
\end{aligned}
$$

Therefore, (A 6) in the Appendix implies $\left\|Q_{j}(t)\right\|_{L_{r^{\prime}}} \leqslant c(\tau), t \in J_{\tau}$, since $\left\|P_{j}(t)\right\|_{L_{\rho}} \leqslant c(\tau)$, $t \in J_{\tau}$, due to the choice of $r$. Here, $\frac{1}{r}+\frac{1}{r^{\prime}}=1$. Since this is true for each $j \in\{1, \ldots, N\}$, assumption $\left(A_{10}\right)$ gives that $\Theta(P, Q)$ belongs to $L_{\infty}\left(J_{\tau}, L_{\rho}\right)$ and hence also $\|m(t)\|_{L_{r^{\prime}}} \leqslant c(\tau)$, $t \in J_{\tau}$. Using Young's inequality and the Gagliardo-Nirenberg inequality [17, p.37] it 
follows analogously to the proof of [30, Prop.5.1] that

$$
\begin{aligned}
& \int_{\Omega} Q_{j}\left(\frac{P_{j}}{\phi_{j}(f)}\right)^{\sigma-1} \mathrm{~d} x+\int_{\Omega} m\left(\frac{P_{j}}{\phi_{j}(f)}\right)^{\sigma} \mathrm{d} x \\
& \leqslant c(\tau, \varepsilon)+c(\tau, \varepsilon) \int_{\Omega}\left(\frac{P_{j}}{\phi_{j}(f)}\right)^{\sigma} \mathrm{d} x+\varepsilon c(\tau) \int_{\Omega}\left|\nabla\left(\frac{P_{j}}{\phi_{j}(f)}\right)^{\sigma / 2}\right|^{2} \mathrm{~d} x,
\end{aligned}
$$

where $\varepsilon>0$ is arbitrary. Applying Young's inequality also for the last term of the right side of (3.34) and choosing $\varepsilon>0$ sufficiently small, we derive

$$
\frac{\mathrm{d}}{\mathrm{d} t} \int_{\Omega} \phi_{j}(f)\left(\frac{P_{j}}{\phi_{j}(f)}\right)^{\sigma} \mathrm{d} x \leqslant c(\tau)+c(\tau) \int_{\Omega}\left(\frac{P_{j}}{\phi_{j}(f)}\right)^{\sigma} \mathrm{d} x+c(\tau) \sum_{l=1}^{N} \int_{\Omega} P_{l}^{\sigma} \mathrm{d} x, \quad t \in \dot{J} .
$$

Summing over all $j=1, \ldots, N$ and using (3.32) the assertion follows from Gronwall's inequality.

Since $n \leqslant 3$ we may, due to (3.29), apply Lemma 3.3 successively to deduce the estimate

$$
\|P(t)\|_{L_{Q}}+\|Q(t)\|_{L_{Q}} \leqslant c(\tau), \quad t \in J_{\tau} .
$$

By considering the interval $J \cap[\varepsilon, \tau]$ for $\varepsilon \in(0, \tau) \cap J$ we may assume without loss of generality that $m^{0} \in W_{\varrho, \mathscr{B}}^{2}$. From (3.35) and $\left(A_{10}\right)$ it follows $\Theta(P, Q)-e m \in L_{\infty}\left(J_{\tau}, L_{\varrho}\right)$ and so $m \in L_{2}\left(J_{\tau}, W_{\varrho, \mathscr{B}}^{2}\right)$ by using maximal regularity for equation (1.15). From Hölder's inequality we thus obtain that

$$
\int_{s}^{t}\|m(\sigma)\|_{W_{Q, \mathscr{B}}^{2}} \mathrm{~d} \sigma \leqslant c(\tau)(t-s)^{1 / 2}, \quad t, s \in J_{\tau}, \quad s \leqslant t .
$$

Therefore,

$$
\|f(t)-f(s)\|_{W_{Q}^{2}} \leqslant c(\tau)|t-s|^{1 / 2}, \quad t, s \in J_{\tau},
$$

and whence, from (3.3),

$$
\left\|A_{\delta}[u(t)]-A_{\delta}[u(s)]\right\|_{\mathscr{L}\left(W_{\varrho, g B}^{2}, L_{\varrho}\right)} \leqslant M|t-s|^{1 / 2}, \quad t, s \in J_{\tau},
$$

for some $M=M(\tau)>0$. The analogous estimate also holds if $\delta$ is replaced by $\gamma$. Thus [3, II.Lem.5.1.3] ensures that, for $2 \vartheta \in(0,2) \backslash\{1+1 / \varrho\}$,

$$
\left\|U_{A_{\delta}[u]}(t, s)\right\|_{\mathscr{L}\left(W_{\varrho, \mathscr{B}}^{29}\right)}+(t-s)^{\vartheta}\left\|U_{A_{\delta}[u]}(t, s)\right\|_{\mathscr{L}\left(L_{\varrho}, W_{\varrho, \mathscr{B}}^{2 \vartheta}\right)} \leqslant c(\tau), \quad t, s \in J_{\tau}, \quad s \leqslant t,
$$

and analogously for $U_{A_{\gamma}[u]}$. Since (3.36) implies $f \in L_{\infty}\left(J_{\tau}, W_{\varrho, \mathscr{B}}^{2}\right)$ we have, due to $\left(A_{9}\right)$,

$$
\left\|R_{4}(u(t))\right\|_{\mathbb{L}_{\varrho}} \leqslant c(\tau)\left(\|p(t)\|_{\mathbb{W}_{\varrho, \mathscr{P}}^{2 \xi}}+\|q(t)\|_{\mathbb{W}_{\varrho, \mathscr{B}}^{2 \xi}}\right), \quad t \in J_{\tau},
$$

and also

$$
\left\|\int_{0}^{\infty} R_{4}(u(t)) \mathrm{d} a\right\|_{L_{\varrho}} \leqslant c(\tau)\left(\|P(t)\|_{W_{\varrho, \mathscr{B}}^{2 \xi}}+\|Q(t)\|_{W_{\varrho, \mathscr{B}}^{2 \xi}}\right), \quad t \in J_{\tau} .
$$


The latter inequality together with (3.25) and (3.38) implies $\|P(t)\|_{W_{Q, \zeta,}^{2 \xi}} \leqslant c(\tau), t \in J_{\tau}$, by Gronwall's inequality and similarly $\|Q(t)\|_{W_{0, \mathscr{B}}^{2 \xi}} \leqslant c(\tau), t \in J_{\tau}$. But then

$$
\|\mathfrak{b}[u(t)]\|_{L_{\infty}\left((0, \infty),\left(W_{0, \mathscr{B}}^{2 \eta}(\Omega)\right)^{N \times N}\right)} \leqslant M, \quad t \in J_{\tau},
$$

for $2 \eta \in(n / \varrho, 2 \xi)$ thanks to Lemma 2.7. From (3.37), (3.40), and Proposition 2.1, we conclude

$$
\left\|\mathscr{U}_{[u]}(t, s)\right\|_{\mathscr{L}\left(\mathbb{L}_{e}, \mathbb{W}_{\varrho, \mathscr{g}}^{2 \xi}\right)} \leqslant c(\tau)(t-s)^{-\xi}, \quad t, s \in J_{\tau}, \quad s<t .
$$

Using (3.39) and Gronwall's inequality we thus infer that $\|p(t)\|_{\mathbb{W}_{\varrho .9}^{2 \xi}} \leqslant c(\tau)$ for $t \in J_{\tau}$ and similarly $\|q(t)\|_{\mathbb{W}_{\varrho, \mathscr{B}}^{2 \xi}} \leqslant c(\tau)$ for $t \in J_{\tau}$. Owing to $P, Q \in L_{\infty}\left(L_{\tau}, W_{\varrho, \mathscr{B}}^{2 \xi}\right) \hookrightarrow L_{\infty}\left(J_{\tau}, L_{\infty}\right)$ and (3.28) there holds $\Gamma(f)+\Lambda(P, Q) \in L_{\infty}\left(J_{\tau}, L_{\infty}\right)$ and hence $w \in L_{\infty}\left(J_{\tau}, W_{\varrho, \mathscr{B}}^{2 \xi}\right)$. Finally, noticing that $\Theta(P, Q) \in L_{\infty}\left(J_{\tau}, W_{\varrho, \mathscr{B}}^{2 \eta}\right)$ by Lemma 2.7 and $\left(A_{4}\right)$, we first obtain $m \in$ $L_{\infty}\left(J_{\tau}, W_{\varrho, \mathscr{B}}^{2 \vartheta}\right)$ for each $\vartheta<1$ from (1.15). Then, since $h \in C^{4 \epsilon}(\bar{\Omega})$ and hence $R_{1}(u) \in$ $L_{\infty}\left(J_{\tau}, W_{\varrho, \mathscr{B}}^{3 \epsilon}\right)$, we also obtain $\|m(t)\|_{W_{\varrho, \mathscr{B}}^{2+2 \epsilon}} \leqslant c(\tau)$ for $t \in J \cap\left[\tau_{0}, \tau\right]$ with $0<\tau_{0}<\tau$ by (2.9). Lemma 2.6 then ensures $f \in L_{\infty}\left(J \cap\left[\tau_{0}, \tau\right], W_{\varrho, \mathscr{B}}^{2+\epsilon}\right)$. Therefore, we conclude (3.22) for each $\tau>0$, whence $J=\mathbb{R}^{+}$. This proves Theorem 3.1.

Remark 3.4 In Subsection 3.2 it was observed that $w, P, Q \in C^{\rho}\left(J, W_{\varrho, \mathscr{B}}^{2 \eta}\right)$ for some $\rho>0$ and $2 \eta \in(n / \varrho, 2)$. Therefore, $b(\cdot, w, P, Q) \in C^{\rho}\left(J, F_{\bar{\eta}}\right)$ for $2 \bar{\eta} \in(n / \varrho, 2 \eta)$ by Lemma 2.7 . Hence classical solutions to equation (1.18) are necessarily mild solutions as noted in the Appendix.

\section{Conclusions}

This paper analyses a mathematical model focusing on key components involved in tumour growth. Tumour cell migration into healthy tissue is due to cell motility and haptotaxis, that is, the directed migratory response of tumour cells up gradients of celladhesion molecules. In addition to spatial position tumour cells are also distinguished by age and mutation type. Thus, the model consists of a system of nonlinear partial differential equations with both parabolic and hyperbolic features. Cell division processes are assumed to be affected by oxygen concentration and total tumour population. This leads to a nonlinear age-boundary condition.

Due to the nonlinear diffusion terms and the nonlinear age-boundary condition the associated abstract linear problem is nonautonomous. It is shown that the abstract formulation yields an evolution system with suitable regularity properties and Lipschitz dependence on the nonlinearities. Based on these properties local existence of unique nonnegative solutions is derived by a fixed point argument. A bootstrapping argument is employed in order to prove that the solutions exist globally in time.

\section{Appendix A}

This appendix is devoted to the proof of Proposition 2.1. We also provide the justification of the definition of the mild solution of $(P)_{s, \phi, g}$ introduced in Definition 2.2. 
For the remainder we fix numbers

$$
\varrho \in(1 \vee n / 2, \infty), \quad 2 \eta \in(n / \varrho, 2) \backslash\{1+1 / \varrho\}, \quad \rho>0, \quad T>0
$$

and put $J_{T}:=[0, T]$. Moreover, we choose

$$
A \in C^{\rho}\left(J_{T}, \mathscr{H}^{+}\left(W_{\varrho, \mathscr{B}}^{2}, L_{\varrho}\right)\right) \quad \text { with } \quad\|A\|_{C^{\rho}\left(J_{T}, \mathscr{H}\left(W_{\varrho, \mathscr{B}}^{2}, L_{\varrho}\right)\right)} \leqslant M
$$

and notice that the corresponding evolution system $\left\{U_{A}(t, s) ;(t, s) \in \Delta_{T}\right\}$ on $\mathbb{L}_{\varrho}$ is positive, that is, the positive cone $\mathbb{L}_{\varrho}^{+}$is invariant under $U_{A}(t, s)$ for each $(t, s) \in \Delta_{T}$. We refer to [3, Chapt.II] for the existence and basic properties of the evolution system $U_{A}$. We also let

$$
\mathfrak{b} \in F_{\eta}=C\left(J_{T}, L_{\infty}^{+}\left((0, \infty),\left(W_{\varrho, \mathscr{B}}^{2 \eta}(\Omega)\right)^{N \times N}\right)\right) \quad \text { with } \quad\|\mathfrak{b}\|_{F_{\eta}} \leqslant M .
$$

Observe that pointwise multiplication

$$
W_{\varrho, \mathscr{B}}^{2 \eta}(\Omega) \times W_{\varrho, \mathscr{B}}^{2 \vartheta}(\Omega) \rightarrow W_{\varrho, \mathscr{B}}^{2 \vartheta}, \quad \vartheta \in\{0, \eta\}
$$

is continuous due to [2, Thm.4.1] since $2 \eta>n / \varrho$. In particular, $\mathfrak{b}(t, a) \in\left(W_{\varrho, \mathscr{B}}^{2 \eta}(\Omega)\right)^{N \times N}$ may be considered as an element of $\mathscr{L}\left(L_{\varrho}\right)$. Next recall that

$$
\left(L_{\varrho}, W_{\varrho, \mathscr{B}}^{2}\right)_{r, \varrho} \doteq W_{\varrho, \mathscr{B}}^{2 r}, \quad 2 r \in(0,2) \backslash\{1,1+1 / \varrho\}, \quad \text { and } \quad\left[L_{\varrho}, W_{\varrho, \mathscr{B}}^{2}\right]_{1 / 2} \doteq W_{\varrho, \mathscr{B}}^{1}
$$

where $(\cdot, \cdot)_{r, \varrho}$ and $[\cdot, \cdot]_{\vartheta}$ denote, respectively, the real and the complex interpolation functor of exponent $r$, and $\doteq$ means "(algebraically) equal with equivalent norms". Then it follows from [3, II.Lem.5.1.3] that

$$
\left\|U_{A}(t, s)\right\|_{\mathscr{L}\left(W_{\varrho, \mathscr{B}}^{2 r}, W_{\varrho, B}^{2 \sigma}\right)} \leqslant c_{T}(M)(t-s)^{\bar{r}-\sigma}, \quad(t, s) \in \Delta_{T}^{*},
$$

for $0 \leqslant \bar{r} \leqslant r \leqslant \sigma \leqslant 1$ with $2 r, 2 \sigma \neq 1+1 / \varrho$ and $\bar{r}<r$ if $0<r<\sigma<1$. Furthermore,

$$
\left\|U_{A}(t, s)\right\|_{\mathscr{L}\left(L_{\varrho}, L_{\xi}\right)} \leqslant c_{T}(M)(t-s)^{-(1 / \varrho-1 / \xi) n / 2}, \quad(t, s) \in \Delta_{T}^{*},
$$

provided $1<\varrho \leqslant \xi \leqslant \infty$.

\section{A.1 Proof of proposition 2.1}

We first focus our attention on the Volterra equation (2.2). We prove the well-posedness of the equation and derive some important properties which then enable us to prove Proposition 2.1.

Lemma A.1 There exists a linear operator $B \in \mathscr{L}\left(\mathbb{L}_{\varrho}, C\left(\Delta_{T}, L_{\varrho}\right)\right)$ such that $B \phi$ is the unique solution of (2.2) for $\phi \in \mathbb{L}_{\varrho}$. More precisely,

$$
\|(B \phi)(t, s)\|_{L_{\varrho}} \leqslant c_{T}(M)\|\phi\|_{\mathbb{L}_{\varrho}}, \quad(t, s) \in \Delta_{T}, \quad \phi \in \mathbb{L}_{\varrho} .
$$

Furthermore, if $\phi \in \mathbb{L}_{\varrho}^{+}$, then $(B \phi)(t, s) \in \mathbb{L}_{\varrho}^{+}$for $(t, s) \in \Delta_{T}$. 
Proof We fix $s \in[0, T)$ and $\phi \in \mathbb{L}_{\varrho}$ and define

$$
z_{s}(\tau):=2 \int_{\tau}^{\infty} \mathfrak{b}(\tau+s, a) U_{A}(\tau+s, s) \phi(a-\tau) \mathrm{d} a, \quad 0 \leqslant \tau \leqslant T-s,
$$

so that $z_{s} \in C\left([0, T-s], L_{\varrho}\right)$ owing to (A 3)-(A 5). Choosing then $\lambda>0$ sufficiently large and putting

$$
F_{S}(v)(\tau):=2 \int_{0}^{\tau} e^{-\lambda a} \mathfrak{b}(\tau+s, a) U_{A}(\tau+s, \tau+s-a) v(\tau-a) \mathrm{d} a+e^{-\lambda \tau} z_{s}(\tau),
$$

it follows that

$$
F_{S}: C\left([0, T-s], L_{\varrho}\right) \rightarrow C\left([0, T-s], L_{\varrho}\right)
$$

is a contraction and thus possesses a unique fixed point $v_{s} \in C\left([0, T-s], L_{\varrho}\right)$. Gronwall's inequality implies

$$
\left\|v_{s}(\tau)\right\|_{L_{\varrho}} \leqslant c_{T}(M)\|\phi\|_{\mathbb{L}_{\varrho}}, \quad 0 \leqslant \tau \leqslant T-s, \quad 0 \leqslant s<T .
$$

Furthermore, if $\phi \in \mathbb{L}_{\varrho}^{+}$, then $z_{s}(\tau) \in L_{\varrho}^{+}$for $\tau \in[0, T-s]$ since $U_{A}$ is positive and so $v_{s}(\tau) \in L_{\varrho}^{+}$for $\tau \in[0, T-s]$. We set

$$
(B \phi)(t, s):=e^{\lambda(t-s)} v_{s}(t-s), \quad(t, s) \in \Delta_{T}, \quad s \neq T,
$$

and

$$
(B \phi)(T, T):=2 \int_{0}^{\infty} \mathfrak{b}(T, a) \phi(a) \mathrm{d} a .
$$

Then $(B \phi)$ solves (2.2). It follows from (A 3), (A 4), (A 8), and the fact that $U_{A}$ is uniformly strongly continuous on compact subsets of $L_{\varrho}$, that $B \phi$ belongs to $C\left(\Delta_{T}, L_{\varrho}\right)$ and satisfies (A 7). The unique solvability of (2.2) in $C\left(\Delta_{T}, L_{\varrho}\right)$ yields that $[\phi \mapsto B \phi]$ is linear, whence $B \in \mathscr{L}\left(\mathbb{L}_{\varrho}, C\left(\Delta_{T}, L_{\varrho}\right)\right)$.

Having established the existence of a unique solution to (2.2) we may now indeed define, for $(t, s) \in \Delta_{T}$, the operator $\mathscr{U}(t, s)=\mathscr{U}_{[A, \mathfrak{b}]}(t, s)$ from $\mathbb{L}_{\varrho}$ into itself by virtue of $(2.1)$. In order to prove that $\mathscr{U}$ defines an evolution operator on $\mathbb{L}_{\varrho}$ we require the following auxiliary result.

Lemma A.2 Given $\phi \in \mathbb{L}_{\varrho}$ we have

$$
(B \mathscr{U}(r, s) \phi)(t, r)=(B \phi)(t, s) \quad \text { for } \quad 0 \leqslant s \leqslant r \leqslant t \leqslant T .
$$

Proof We may assume that $0 \leqslant s<r \leqslant t \leqslant T$ since $\mathscr{U}(s, s) \phi=\phi$. We observe that

$$
\begin{aligned}
(B \mathscr{U}(r, s) \phi)(t, r)= & 2 \int_{0}^{t-r} \mathfrak{b}(t, a) U_{A}(t, t-a)(B \mathscr{U}(r, s) \phi)(t-a, r) \mathrm{d} a \\
& +2 \int_{t-r}^{t-s} \mathfrak{b}(t, a) U_{A}(t, t-a)(B \phi)(t-a, s) \mathrm{d} a \\
& +2 \int_{t-s}^{\infty} \mathfrak{b}(t, a) U_{A}(t, s) \phi(a-t+s) \mathrm{d} a
\end{aligned}
$$


Therefore, setting

$$
g(t):= \begin{cases}(B \mathscr{U}(r, s) \phi)(t, r), & r \leqslant t \leqslant T \\ (B \phi)(t, s), & s \leqslant t<r\end{cases}
$$

we deduce, for $t \in[s, T]$, that

$$
g(t)=2 \int_{0}^{t-s} \mathfrak{b}(t, a) U_{A}(t, t-a) g(t-a) \mathrm{d} a+2 \int_{t-s}^{\infty} \mathfrak{b}(t, a) U_{A}(t, s) \phi(a-t+s) \mathrm{d} a .
$$

Clearly, $g(r)=\lim _{t \rightarrow r^{-}} g(t)$ by (2.2), (2.3), and $B \phi \in C\left(\Delta_{T}, L_{\varrho}\right)$, whence $g \in C\left([s, T], L_{\varrho}\right)$. The uniqueness statement of Lemma A.1 thus gives that $g(t)=(B \phi)(t, s)$ for $s \leqslant t \leqslant T$, from which the assertion follows.

We are now in a position to prove Proposition 2.1. We first show that (2.1)-(2.2) indeed define an evolution operator on $\mathbb{L}_{\varrho}$. Note that it immediately follows from Lemma A.1 and (2.1)-(2.2) that $\mathscr{U}(t, s) \in \mathscr{L}\left(\mathbb{L}_{\varrho}\right)$ for $(t, s) \in \Delta_{T}$ with

$$
\|\mathscr{U}(t, s) \phi\|_{\mathbb{L}_{\varrho}} \leqslant c_{T}(M)\|\phi\|_{\mathbb{L}_{\varrho}}, \quad(t, s) \in \Delta_{T},
$$

and that $\mathscr{U}(t, s) \phi \in \mathbb{L}_{\varrho}^{+}$provided $\phi \in \mathbb{L}_{\varrho}^{+}$. Next, let $0 \leqslant s<r \leqslant t \leqslant T$ and $\phi \in \mathbb{L}_{\varrho}$. Then, using (2.1) and the fact that $U_{A}$ is an evolution operator, we derive, for a.a. $a>0$, that

$$
\begin{aligned}
{[\mathscr{U}(t, r) \mathscr{U}(r, s) \phi](a) } & = \begin{cases}U_{A}(t, t-a)(B \mathscr{U}(r, s) \phi)(t-a, r), & 0 \leqslant a \leqslant t-r, \\
U_{A}(t, t-a)(B \phi)(t-a, s), & t-r<a \leqslant t-s, \\
U_{A}(t, s) \phi(a-t+s), & t-s<a,\end{cases} \\
& =[\mathscr{U}(t, s) \phi](a),
\end{aligned}
$$

where we invoked Lemma A.2 for the last equality. Therefore, parts (i)-(iii) of Proposition 2.1 are proved.

Next we prove part (iv) of Proposition 2.1. Let $\phi \in \mathbb{W}_{\varrho, \mathscr{B}}^{2 \theta}$ with $2 \theta \in(0,2) \backslash\{1+1 / \varrho\}$, and let $(t, s),(\bar{t}, \bar{s}) \in \Delta_{T}$, where we may assume that $t-s \leqslant \bar{t}-\bar{s}$. Then, from (A 5) and (A 7),

$$
\begin{aligned}
& \|\mathscr{U}(t, s) \phi-\mathscr{U}(\bar{t}, \bar{s}) \phi\|_{\mathbb{W}_{\varrho, \mathscr{B}}^{2 \theta}} \\
& \leqslant \int_{0}^{t-s}\left\|U_{A}(t, t-a)\right\|_{\mathscr{L}\left(L_{\varrho}, W_{\varrho, O}^{2 \theta}\right)}\|(B \phi)(t-a, s)-(B \phi)(\bar{t}-a, \bar{s})\|_{L_{\varrho}} \mathrm{d} a \\
& +\int_{0}^{t-s}\left\|\left[U_{A}(t, t-a)-U_{A}(\bar{t}, \bar{t}-a)\right](B \phi)(\bar{t}-a, \bar{s})\right\|_{W_{\varrho, g}^{2 \theta}} \mathrm{d} a \\
& +\int_{t-s}^{\bar{t}-\bar{s}}\left\|U_{A}(t, s)\right\|_{\mathscr{L}\left(W_{\varrho, \mathscr{B}}^{2 \theta}\right)}\|\phi(a-t+s)\|_{W_{\varrho, \mathscr{B}}^{2 \theta}} \mathrm{d} a \\
& +\int_{t-S}^{\bar{t}-\bar{s}}\left\|U_{A}(\bar{t}, \bar{t}-a)\right\|_{\mathscr{L}\left(L_{\varrho}, W_{Q, \mathscr{B}}^{2 \theta}\right)}\|(B \phi)(\bar{t}-a, \bar{s})\|_{L_{\varrho}} \mathrm{d} a \\
& +\int_{\bar{t}-\bar{s}}^{\infty}\left\|U_{A}(t, s) \phi(a-t+s)-U_{A}(\bar{t}, \bar{s}) \phi(a-\bar{t}+\bar{s})\right\|_{W_{0, B}^{2 \theta}} \mathrm{d} a
\end{aligned}
$$




$$
\begin{aligned}
\leqslant & c_{T}(M) \int_{0}^{t-s} a^{-\theta}\|(B \phi)(t-a, s)-(B \phi)(\bar{t}-a, \bar{s})\|_{L_{\varrho}} \mathrm{d} a \\
& +\int_{0}^{t-s}\left\|\left[U_{A}(t, t-a)-U_{A}(\bar{t}, \bar{t}-a)\right](B \phi)(\bar{t}-a, \bar{s})\right\|_{W_{Q, 9}^{2 \theta}} \mathrm{d} a \\
& +c_{T}(M) \int_{0}^{\bar{t}-\bar{s}-(t-s)}\|\phi(a)\|_{W_{Q, Q}^{2 \theta}} \mathrm{d} a+c_{T}(M)\|\phi\|_{\mathbb{L}_{\varrho}} \int_{t-s}^{\bar{t}-\bar{s}} a^{-\theta} \mathrm{d} a \\
& +c_{T}(M) \int_{\bar{t}-\bar{s}}^{\infty}\|\phi(a-t+s)-\phi(a-\bar{t}+\bar{s})\|_{W_{Q, Q}^{2 \theta}} \mathrm{d} a \\
& +\int_{\bar{t}-\bar{s}}^{\infty}\left\|\left[U_{A}(t, s)-U_{A}(\bar{t}, \bar{s})\right] \phi(a-\bar{t}+\bar{s})\right\|_{W_{Q, Q}^{2 \theta}} \mathrm{d} a .
\end{aligned}
$$

That the right side tends to zero as $|t-\bar{t}|+|s-\bar{s}| \rightarrow 0$ now follows from $B \phi \in C\left(\Delta_{T}, L_{\varrho}\right)$, the fact that translations are strongly continuous on $\mathbb{W}_{\varrho, \mathscr{B}}^{2 \theta}$, and the fact that $U_{A}$ is uniformly strongly continuous on compact subsets of $W_{\varrho, \mathscr{B}}^{20}$. For the second integral one may want to use an approximation of $B \phi \in C\left(\Delta_{T}, L_{\varrho}\right)$ by functions in $C\left(\Delta_{T}, W_{\varrho, \mathscr{B}}^{2 \theta}\right)$, and for the last integral one may also want to use that the compactly supported continuous functions from $(0, \infty)$ into $W_{\varrho, \mathscr{B}}^{2 \theta}$ are dense in $\mathbb{W}_{\varrho, \mathscr{B}}^{2 \theta}$. Therefore, one concludes that $[(t, s) \mapsto \mathscr{U}(t, s) \phi] \in C\left(\Delta_{T}, \mathbb{W}_{\varrho, \mathscr{B}}^{2 \theta}\right)$.

As for (2.6), let $0 \leqslant \bar{\theta} \leqslant \theta \leqslant \sigma \leqslant 1$ with $2 \theta, 2 \sigma \neq 1+1 / \varrho$ and $\bar{\theta}<\theta$ if $0<\theta<\sigma<1$. Let $\phi \in \mathbb{W}_{\varrho, \mathscr{B}}^{2 \theta}$ so that $B \phi \in C\left(\Delta_{T}, L_{\varrho}\right)$. Put $\vartheta:=\bar{\theta}$ if $\theta \leqslant \eta$ and $\vartheta:=\eta$ if $\eta<\theta$. We choose $(t, s) \in \Delta_{T}^{*}$ and notice that from (A 3)-(A 5) and (2.2)

$$
\begin{aligned}
\|(B \phi)(t, s)\|_{W_{\varrho, \mathscr{B}}^{2 \eta}} \leqslant & 2 \int_{0}^{t-s}\|\mathfrak{b}(t, a)\|_{\mathscr{L}\left(W_{\varrho, \mathscr{B}}^{2 \eta}\right)}\left\|U_{A}(t, t-a)\right\|_{\mathscr{L}\left(L_{\varrho}, W_{\varrho, \mathscr{B}}^{2 \eta}\right)}\|(B \phi)(t-a, s)\|_{L_{\varrho}} \mathrm{d} a \\
& +2 \int_{t-s}^{\infty}\|\mathfrak{b}(t, a)\|_{\mathscr{L}\left(W_{\varrho, \mathscr{B}}^{2 \eta}\right)}\left\|U_{A}(t, s)\right\|_{\mathscr{L}\left(W_{\varrho, \mathscr{B}}^{2 \theta}, W_{\varrho, \mathscr{S}}^{2 \eta}\right)}\|\phi(a-t+s)\|_{W_{\varrho, \mathscr{B}}^{2 \theta}} \mathrm{d} a \\
\leqslant & c_{T}(M)(t-s)^{1-\eta}\|\phi\|_{\mathbb{L}_{\varrho}}+c_{T}(M)(t-s)^{\vartheta-\eta}\|\phi\|_{\mathbb{W}_{\varrho, \mathscr{P}}^{2 \theta}}
\end{aligned}
$$

and therefore

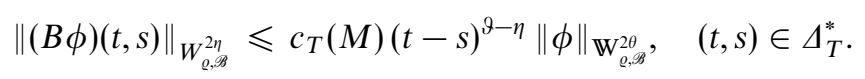

Choosing $\bar{\eta} \in(0, \eta)$ if $\eta<\sigma$ and setting $\bar{\eta}:=\sigma$ if $\sigma \leqslant \eta$ we infer from (A 5), (A 10), and Lemma A.1 that

$$
\begin{aligned}
& \|\mathscr{U}(t, s) \phi\|_{\mathbb{W}_{\varrho, \mathscr{B}}^{2 \sigma}} \leqslant \int_{0}^{t-s}\left\|U_{A}(t, t-a)\right\|_{\mathscr{L}\left(W_{Q, \mathscr{B}}^{2 \eta}, W_{Q, \mathscr{B}}^{2 \sigma}\right)}\|(B \phi)(t-a, s)\|_{W_{\varrho, \mathscr{B}}^{2 \eta}} \mathrm{d} a \\
& +\int_{t-s}^{\infty}\left\|U_{A}(t, s)\right\|_{\mathscr{L}\left(W_{\varrho, \mathscr{S}}^{2 \theta}, W_{\varrho, \mathscr{Q}}^{2 \sigma}\right)}\|\phi(a-t+s)\|_{W_{Q, \mathscr{O}}^{2 \theta}} \mathrm{d} a \\
& \leqslant c_{T}(M)\left\{\int_{0}^{t-s} a^{\bar{\eta}-\sigma}(t-s-a)^{\vartheta-\eta} \mathrm{d} a+c_{T}(M)(t-s)^{\bar{\theta}-\sigma}\right\}\|\phi\|_{W_{\varrho, \mathscr{B}}^{2 \theta}} .
\end{aligned}
$$

Observing that

$$
\int_{0}^{t-s} a^{-\xi}(t-s-a)^{-\zeta} \mathrm{d} a=(t-s)^{1-\xi-\zeta} \mathrm{B}(1-\xi, 1-\zeta), \quad \xi, \zeta<1,
$$

with B denoting the beta function, we deduce (2.6). 
To finish the proof of Proposition 2.1 it remains to show that the evolution system depends Lipschitz continuously on the data $A$ and $\mathfrak{b}$. Thus assume that $A$ and $\bar{A}$ both satisfy (2.4) and that $\mathfrak{b}$ and $\overline{\mathfrak{b}}$ both satisfy (2.5). Put

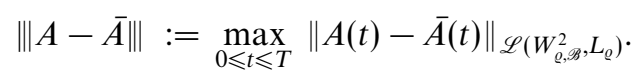

In the following we write

$$
\mathscr{U}:=\mathscr{U}_{[A, \mathfrak{b}]}, \quad \overline{\mathscr{U}}:=\mathscr{U}_{[\bar{A}, \overline{\mathfrak{b}}]}, \quad B:=B_{[A, \mathfrak{b}]}, \quad \bar{B}:=B_{[\bar{A}, \overline{\mathfrak{b}}]} .
$$

Given $2 \theta \in(0,2] \backslash\{1+1 / \varrho\}$ and $2 \sigma \in[0,2) \backslash\{1+1 / \varrho\}$ we choose $\bar{\theta} \in(0, \theta)$ and $\bar{\eta} \in(0, \eta)$ such that

$$
\theta-\bar{\theta}+\eta-\bar{\eta}<1
$$

As above we set $\vartheta:=\bar{\theta}$ if $\theta \leqslant \eta$ and $\vartheta:=\eta$ if $\eta<\theta$. Recall then that

$$
\left\|U_{A}(t, s)-U_{\bar{A}}(t, s)\right\|_{\mathscr{L}\left(W_{Q, \mathscr{B}}^{2 \xi}, W_{Q, \mathscr{B}}^{2 \tau}\right)} \leqslant c_{T}(M)(t-s)^{\xi-\tau}\|A-\bar{A}\|, \quad(t, s) \in \Delta_{T}^{*},
$$

for $2 \xi \in(0,2] \backslash\{1+1 / \varrho\}$ and $2 \tau \in[0,2) \backslash\{1+1 / \varrho\}$ according to [3, II.Lem.5.1.4]. Invoking (A 4), (A 5), (A 7), (A 10), and (A 13) we derive, for $\phi \in \mathbb{W}_{\varrho, \mathscr{B}}^{2 \theta}$ and $(t, s) \in \Delta_{T}^{*}$,

$$
\begin{aligned}
& \|(B \phi)(t, s)-(\bar{B} \phi)(t, s)\|_{W_{Q, Q B S}^{2 \eta}} \\
& \leqslant 2 \int_{0}^{t-s}\|\mathfrak{b}(t, a)-\overline{\mathfrak{b}}(t, a)\|_{\mathscr{L}\left(W_{\varrho, \mathscr{B}}^{2 \eta}\right)}\left\|U_{A}(t, t-a)\right\|_{\mathscr{L}\left(L_{\varrho}, W_{Q, Q B}^{2 \eta}\right)}\|(B \phi)(t-a, s)\|_{L_{Q}} \mathrm{~d} a \\
& +2 \int_{0}^{t-s}\|\overline{\mathfrak{b}}(t, a)\|_{\mathscr{L}\left(W_{\varrho, \mathscr{B}}^{2 \eta}\right)}\left\|U_{A}(t, t-a)-U_{\bar{A}}(t, t-a)\right\|_{\mathscr{L}\left(W_{\varrho, \mathscr{B}}^{2 \eta}\right)}\|(B \phi)(t-a, s)\|_{W_{\varrho, \mathscr{B}}^{2 \eta}} \mathrm{d} a \\
& +2 \int_{0}^{t-s}\|\overline{\mathfrak{b}}(t, a)\|_{\mathscr{L}\left(W_{\varrho, B}^{2 \eta}\right)}\left\|U_{\bar{A}}(t, t-a)\right\|_{\mathscr{L}\left(W_{Q, \mathscr{B}}^{2 \eta}\right)}\|(B \phi)(t-a, s)-(\bar{B} \phi)(t-a, s)\|_{W_{\varrho, \mathscr{B}}^{2 \eta}} \mathrm{d} a \\
& \left.+2 \int_{t-s}^{\infty} \| \mathfrak{b}(t, a)-\overline{\mathfrak{b}}(t, a)\right)\left\|_{\mathscr{L}\left(W_{\varrho, g}^{2 \eta}\right)}\right\| U_{A}(t, s)\left\|_{\mathscr{L}\left(W_{\varrho, \mathscr{B}}^{2 \theta}, W_{\varrho, \mathscr{B}}^{2 \eta}\right)}\right\| \phi(a-t+s) \|_{W_{\varrho, \mathscr{B}}^{2 \theta}} \mathrm{d} a \\
& \left.+2 \int_{t-s}^{\infty} \| \overline{\mathfrak{b}}(t, a)\right)\left\|_{\mathscr{L}\left(W_{\varrho, B}^{2 \eta}\right)}\right\| U_{A}(t, s)-U_{\bar{A}}(t, s)\left\|_{\mathscr{L}\left(W_{\varrho, B}^{2 \theta}, W_{\varrho, \mathscr{B}}^{2 \eta}\right)}\right\| \phi(a-t+s) \|_{W_{\varrho, B}^{2 \theta}} \mathrm{d} a \\
& \leqslant c_{T}(M)\|\mathfrak{b}-\overline{\mathfrak{b}}\|_{F_{\eta}}(t-s)^{1-\eta}\|\phi\|_{\mathbb{L}_{\varrho}}+c_{T}(M)\|A-\bar{A}\|(t-s)^{1+\vartheta-\eta}\|\phi\|_{\mathbb{W}_{\varrho, \mathscr{O}}^{2 \theta}} \\
& +c_{T}(M) \int_{0}^{t-s}\|(B \phi)(t-a, s)-(\bar{B} \phi)(t-a, s)\|_{W_{0,9}^{2 \eta}} \mathrm{d} a \\
& +c_{T}(M)\|\mathfrak{b}-\overline{\mathfrak{b}}\|_{F_{\eta}}(t-s)^{\bar{\theta}-\eta}\|\phi\|_{\mathbb{W}_{\varrho, Q S}^{2 \theta}}+c_{T}(M)\|A-\bar{A}\|(t-s)^{\theta-\eta}\|\phi\|_{\mathbb{W}_{\varrho, \mathscr{B}}^{2 \theta}} .
\end{aligned}
$$

Since $0<\bar{\theta}<\theta \leqslant 1$, the singular Gronwall inequality [3, II.Thm.3.3.1] yields

$$
\|(B \phi)(t, s)-(\bar{B} \phi)(t, s)\|_{W_{\varrho, B}^{2 \eta}} \leqslant c_{T}(M)(t-s)^{\bar{\theta}-\eta}\left[\|\mathfrak{b}-\overline{\mathfrak{b}}\|_{F_{\eta}}+\|A-\bar{A}\| \|\right], \quad(t, s) \in \Delta_{T}^{*} .
$$


Using this estimate together with (A 5), (A 10), and (A 13) we derive from (2.1) that, for $(t, s) \in \Delta_{T}^{*}$,

$$
\begin{aligned}
& \|\mathscr{U}(t, s) \phi-\overline{\mathscr{U}}(t, s) \phi\|_{\mathbb{W}_{\varrho, \mathscr{B}}^{2 \sigma}} \\
& \leqslant \int_{0}^{t-s}\left\|U_{A}(t, t-a)-U_{\bar{A}}(t, t-a)\right\|_{\mathscr{L}\left(W_{Q, \mathscr{B}}^{2 n}, W_{\varrho, \mathscr{B}}^{2 \sigma}\right)}\|(B \phi)(t-a, s)\|_{W_{Q, \mathscr{B}}^{2 n}} \mathrm{~d} a \\
& +\int_{0}^{t-s}\left\|U_{\bar{A}}(t, t-a)\right\|_{\mathscr{L}\left(W_{Q, B}^{2 \eta}, W_{Q, B}^{2 \sigma}\right)}\|(B \phi)(t-a, s)-(\bar{B} \phi)(t-a, s)\|_{W_{Q, \mathscr{B}}^{2 \eta}} \mathrm{d} a \\
& +\int_{t-s}^{\infty}\left\|U_{A}(t, s)-U_{\bar{A}}(t, s)\right\|_{\mathscr{L}\left(W_{\varrho, \mathscr{B}}^{2 \theta}, W_{\varrho, \mathscr{B}}^{2 \sigma}\right)}\|\phi(a-t+s)\|_{W_{\varrho, \mathscr{B}}^{2 \theta}} \mathrm{d} a \\
& \leqslant c_{T}(M)\|A-\bar{A}\|\|\phi\|_{\mathbb{W}_{\varrho, \mathscr{B}}^{2 \theta}} \int_{0}^{t-s} a^{\eta-\sigma}(t-s-a)^{\vartheta-\eta} \mathrm{d} a \\
& +c_{T}(M)\|\phi\|_{\mathbb{W}_{\varrho, \mathscr{B}}^{2 \theta}}\left[\|\mathfrak{b}-\overline{\mathfrak{b}}\|_{F_{\eta}}+\|A-\bar{A}\|\right] \int_{0}^{t-s} a^{\bar{\eta}-\sigma}(t-s-a)^{\bar{\theta}-\eta} \mathrm{d} a \\
& +c_{T}(M)\|A-\bar{A}\|\|\phi\|_{\mathbb{W}_{\varrho, \mathscr{B}}^{2 \theta}}(t-s)^{\theta-\sigma} .
\end{aligned}
$$

We then use (A 11) and (A 12) in order to conclude that

$$
\|\mathscr{U}(t, s) \phi-\overline{\mathscr{U}}(t, s) \phi\|_{\mathbb{W}_{\varrho, \mathscr{B}}^{2 \sigma}} \leqslant c_{T}(M)(t-s)^{\theta-\sigma}\left[\|\mathfrak{b}-\overline{\mathfrak{b}}\|_{F_{\eta}}+\|A-\bar{A}\|\right]\|\phi\|_{\mathbb{W}_{\varrho, \mathscr{B}}^{2 \theta}}
$$

for $(t, s) \in \Delta_{T}^{*}$. Thus, the proof of Proposition 2.1 is complete.

The following lemma was used for the positivity in Lemma 2.4.

Lemma A.3 Suppose $A$ and $\mathfrak{b}$ satisfy (A 1)-(A 3). For $\omega \in \mathbb{R}$ put $A_{\omega}:=A+\omega$. Then

$$
\mathscr{U}_{\left[A_{\omega}, \mathfrak{b}\right]}(t, s)=e^{-\omega(t-s)} \mathscr{U}_{[A, \mathfrak{b}]}(t, s), \quad(t, s) \in \Delta_{T} .
$$

Proof Let $B=B_{[A, \mathfrak{b}]}$ and $B_{\omega}:=B_{\left[A_{\omega}, \mathfrak{b}\right]}$. Choose any $\phi \in \mathbb{L}_{\varrho}$. Then, due to the fact that $U_{A_{\omega}}(t, s)=e^{-\omega(t-s)} U_{A}(t, s)$ for $(t, s) \in \Delta_{T}$, the function $R \in C\left(\Delta_{T}, L_{\varrho}\right)$, defined as

$$
R(t, s):=e^{-\omega(t-s)}(B \phi)(t, s), \quad(t, s) \in \Delta_{T},
$$

satisfies the Volterra equation

$$
R(t, s)=2 \int_{0}^{t-s} \mathfrak{b}(t, a) U_{A_{\omega}}(t, t-a) R(t-a, s) \mathrm{d} a+2 \int_{t-s}^{\infty} \mathfrak{b}(t, a) U_{A_{\omega}}(t, s) \phi(a-t+s) \mathrm{d} a,
$$

for $(t, s) \in \Delta_{T}$, hence $R=B_{\omega} \phi$ on $\Delta_{T}$ by Lemma A.1. This readily implies the assertion according to (2.1).

\section{A.2 Mild solutions to $(P)_{s, \phi, g}$}

Still supposing that $A$ and $\mathfrak{b}$ satisfy (A 1)-(A 3), we now characterize the generator of the evolution operator $\mathscr{U}=\mathscr{U}_{[A, \mathfrak{b}]}$. This will be the basis for the justification of the definition of mild solutions. 
Let $s \in[0, T)$ be fixed and define

$$
\left[V_{s}(t) \phi\right](a):=\left\{\begin{array}{ll}
e^{-t A(s)} \phi(a-t), & 0 \leqslant t<a \\
e^{-a A(s)} H_{\phi}^{s}(t-a), & 0 \leqslant a \leqslant t
\end{array}, \quad \phi \in \mathbb{L}_{\varrho},\right.
$$

where $\left\{e^{-t A(s)} ; t \geqslant 0\right\}$ denotes the analytic semigroup on $L_{\varrho}$ generated by $-A(s)$ and where $H_{\phi}^{s} \in C\left(\mathbb{R}^{+}, L_{\varrho}\right)$ satisfies the linear Volterra equation

$$
H_{\phi}^{s}(t)=2 \int_{0}^{t} \mathfrak{b}(s, a) e^{-a A(s)} H_{\phi}^{s}(t-a) \mathrm{d} a+2 \int_{t}^{\infty} \mathfrak{b}(s, a) e^{-t A(s)} \phi(a) \mathrm{d} a, \quad t \geqslant 0 .
$$

It is straightforward to generalize [29, Prop.2.2] to show that (A 14) defines a strongly continuous positive semigroup $\left\{V_{s}(t) ; t \geqslant 0\right\}$ on $\mathbb{L}_{\varrho}$ (in [29] the case $N=1$ and $A(s)=$ $-\Delta_{x}$ was considered with $\mathfrak{b}(s, \cdot) \in C\left(\mathbb{R}^{+}\right)$bounded $)$. Denoting by $-\mathbb{A}(s)$ the generator of $\left\{V_{s}(t) ; t \geqslant 0\right\}$, i.e. $V_{s}(t)=e^{-t \mathbb{A}(s)}, t \geqslant 0$, and by $D(\mathbb{A}(s))$ its domain, it was shown in [29, Prop.2.2] that

$$
\mathbb{A}(s) \phi=\left(\partial_{a}+A(s)\right) \phi, \quad \phi \in D(\mathbb{A}(s))
$$

However, the domain is not known explicitly and rather a core for $\mathbb{A}(s)$ was given and shown that

$$
D(\mathbb{A}(s)) \subset \mathbb{W}_{\varrho, \mathscr{B}}^{2 \theta} \cap W_{1}^{\theta}\left((0, \infty), L_{\varrho}\right), \quad \theta<1 .
$$

The next proposition implies together with (A 16) that the evolution operator $\mathscr{U}=\mathscr{U}_{[A, \mathfrak{b}]}$ is indeed the one corresponding to problem $(P)_{s, \phi, 0}$.

Proposition A.1 Suppose in addition to (A 1)-(A 3) that $\mathfrak{b} \in C^{\rho}\left(J_{T}, F_{\eta}\right)$. For $s \in[0, T)$, let $-\mathbb{A}(s)$ be the generator of the semigroup $\left\{V_{s}(t) ; t \geqslant 0\right\}$ defined in (A 14). Then

(i) $\left.\frac{\partial^{+}}{\partial t} \mathscr{U}(t, s) \phi\right|_{t=s}=-\mathbb{A}(s) \phi, \quad \phi \in D(\mathbb{A}(s)) \cap \mathbb{W}_{\varrho, \mathscr{B}}^{2}, \quad s \in[0, T)$,

(ii) $\frac{\partial}{\partial s} \mathscr{U}(t, s) \phi=\mathscr{U}(t, s) \mathbb{A}(s) \phi, \quad \phi \in D(\mathbb{A}(s)) \cap \mathbb{W}_{\varrho, \mathscr{B}}^{2}, \quad(t, s) \in \Delta_{T}^{*}$.

Proof The proof follows basically [24, 5.Thm.3.1]. Let $(t, s) \in \Delta_{T}^{*}$ and $\phi \in \mathbb{W}_{\varrho, \mathscr{B}}^{2}$. Note that, given $\tau \in[0, T]$, we have

$$
H_{\phi}^{\tau}(t)=2 \int_{0}^{\infty} \mathfrak{b}(\tau, a)\left[e^{-t \mathbb{A}(\tau)} \phi\right](a) \mathrm{d} a,
$$

due to (A 14)-(A 15). Using (2.1) and (A 14) we first compute that

$$
\begin{aligned}
\left\|\mathscr{U}(t, s) \phi-e^{-(t-s) \mathbb{A}(\tau)} \phi\right\|_{\mathbb{L}_{\varrho} \leqslant} & \int_{0}^{t-s}\left\|\left[U_{A}(t, t-a)-e^{-a A(\tau)}\right](B \phi)(t-a, s)\right\|_{L_{\varrho}} \mathrm{d} a \\
& +c_{0} \int_{0}^{t-s}\left\|(B \phi)(t-a, s)-H_{\phi}^{\tau}(t-s-a)\right\|_{L_{\varrho}} \mathrm{d} a \\
& +\int_{t-s}^{\infty}\left\|\left[U_{A}(t, s)-e^{-(t-s) A(\tau)}\right] \phi(a-t+s)\right\|_{L_{\varrho}} \mathrm{d} a
\end{aligned}
$$


where $c_{0}:=\max _{0 \leqslant a, r \leqslant T}\left\|e^{-a A(r)}\right\|_{\mathscr{L}\left(L_{\ell}\right)}$. Next observe that (2.3) and (A 17) allow us to estimate the second integral on the right side by

$$
\begin{aligned}
& 2 c_{0} \int_{0}^{t-s} \int_{0}^{\infty}\|\mathfrak{b}(t-a, z)-\mathfrak{b}(\tau, z)\|_{L_{\infty}\left(\Omega, \mathbb{R}^{N \times N}\right)}\|[\mathscr{U}(t-a, s) \phi](z)\|_{L_{\varrho}} \mathrm{d} z \mathrm{~d} a \\
& \quad+2 c_{0} \int_{0}^{t-s} \int_{0}^{\infty}\|\mathfrak{b}(\tau, z)\|_{L_{\infty}\left(\Omega, \mathbb{R}^{N \times N}\right)}\left\|[\mathscr{U}(t-a, s) \phi](z)-\left[e^{-(t-s-a) \mathbb{A}(\tau)} \phi\right](z)\right\|_{L_{\varrho}} \mathrm{d} z \mathrm{~d} a
\end{aligned}
$$

and thus, taking into account (A 3) and (A 9), further by $c_{T}\left(M,\|b\|_{C^{\rho}}\right) \int_{0}^{t-s}|t-\tau-a|^{\rho} \mathrm{d} a\|\phi\|_{\mathbb{L}_{\varrho}}+c_{T}(M) \int_{0}^{t-s}\left\|\mathscr{U}(t-a, s) \phi-e^{-(t-s-a) \mathbb{A}(\tau)} \phi\right\|_{\mathbb{L}_{\varrho}} \mathrm{d} a$.

Therefore, we obtain

$$
\begin{aligned}
\left\|\mathscr{U}(t, s) \phi-e^{-(t-s) \mathbb{A}(\tau)} \phi\right\|_{\mathbb{L}_{\varrho}} \leqslant & \int_{0}^{t-s}\left\|\left[U_{A}(t, t-a)-e^{-a A(\tau)}\right](B \phi)(t-a, s)\right\|_{L_{\varrho}} \mathrm{d} a \\
& +c_{T}\left(M,\|b\|_{C^{\rho}}\right) \int_{0}^{t-s}|t-\tau-a|^{\rho} \mathrm{d} a\|\phi\|_{\mathbb{L}_{\varrho}} \\
& +c_{T}(M) \int_{0}^{t-s}\left\|\mathscr{U}(t-a, s) \phi-e^{-(t-s-a) \mathbb{A}(\tau)} \phi\right\|_{\mathbb{L}_{\varrho}} \mathrm{d} a \\
& +\int_{0}^{\infty}\left\|\left[U_{A}(t, s)-e^{-(t-s) A(\tau)}\right] \phi(a)\right\|_{L_{\varrho}} \mathrm{d} a \\
= & : I_{1}(t, s)+I_{2}(t, s)+I_{3}(t, s)+I_{4}(t, s) .
\end{aligned}
$$

To prove (i) we now take $\tau=s \in[0, T)$ in (A 18). Since the integrand in $I_{1}(t, s)$ is continuous with respect to $a \in[0, t-s]$ thanks to Lemma A.1, we have

$$
\lim _{t \rightarrow s^{+}} \frac{1}{t-s} I_{1}(t, s)=0 .
$$

Similarly, Proposition 2.1 (iv) ensures

$$
\lim _{t \rightarrow s^{+}} \frac{1}{t-s} I_{3}(t, s)=0 .
$$

Next, since $\phi \in \mathbb{W}_{\varrho, \mathscr{B}}^{2}$, that is, $\phi(a) \in W_{\varrho, \mathscr{B}}^{2}$ for a.a $a>0$, we derive from equality (2.2.2) on page 47 in [3] and (A 2), (A 5) that

$$
\begin{aligned}
\| U_{A}(t, s) \phi(a)- & e^{-(t-s) A(s)} \phi(a) \|_{L_{\varrho}} \\
& \leqslant \int_{s}^{t}\left\|U_{A}(t, r)\right\|_{\mathscr{L}\left(L_{\varrho}\right)}\|A(r)-A(s)\|_{\mathscr{L}\left(W_{Q, \mathscr{B}}^{2}, L_{\varrho}\right)}\left\|e^{-(r-s) A(s)} \phi(a)\right\|_{W_{\varrho, \mathscr{B}}^{2}} \mathrm{~d} r \\
& \leqslant c_{T}(M) \int_{S}^{t}(r-s)^{\rho} \mathrm{d} r\|\phi(a)\|_{W_{\varrho, \mathscr{B}}^{2}}
\end{aligned}
$$

for a.a $a>0$ and so

$$
\lim _{t \rightarrow s^{+}} \frac{1}{t-s} I_{4}(t, s)=0
$$


Therefore, (A 18)-(A 22) yield

$$
\lim _{t \rightarrow s^{+}} \frac{1}{t-s}\left\|\mathscr{U}(t, s) \phi-e^{-(t-s) \mathbb{A}(s)} \phi\right\|_{\mathbb{L}_{\varrho}}=0 .
$$

If $\phi \in D(\mathbb{A}(s))$, then, since $\left\{e^{-t \mathbb{A}(s)} ; t \geqslant 0\right\}$ is a strongly continuous semigroup on $\mathbb{L}_{\varrho}$, we also have

$$
\left.\frac{\partial^{+}}{\partial t} e^{-(t-s) \mathbb{A}(s)} \phi\right|_{t=s}=-\mathbb{A}(s) \phi \quad \text { in } \mathbb{L}_{\varrho} .
$$

Combining (A 23) and (A 24) we obtain part (i) of the assertion. In order to prove part (ii) we take $\tau=t$ in (A 18) and use equality (2.2.3) on page 48 in [3] for the analogue of (A 21). We thus deduce similarly as above that

$$
\lim _{s \rightarrow t^{-}} \frac{1}{t-s}\left\|\mathscr{U}(t, s) \phi-e^{-(t-s) \mathbb{A}(t)} \phi\right\|_{\mathbb{L}_{\varrho}}=0, \quad t \in(0, T], \quad \phi \in \mathbb{W}_{\varrho, \mathscr{B}}^{2},
$$

from which we conclude, owing to

$$
\left.\frac{\partial^{-}}{\partial s} e^{-(t-s) \mathbb{A}(s)} \psi\right|_{s=t}=\mathbb{A}(t) \psi, \quad \psi \in D(\mathbb{A}(t))
$$

that

$$
\left.\frac{\partial^{-}}{\partial s} \mathscr{U}(t, s) \psi\right|_{s=t}=\mathbb{A}(t) \psi, \quad t \in(0, T], \quad \psi \in \mathbb{W}_{\varrho, \mathscr{B}}^{2} \cap D(\mathbb{A}(t)) .
$$

If $(t, s) \in \Delta_{T}$ and $\phi \in \mathbb{W}_{\varrho, \mathscr{B}}^{2} \cap D(\mathbb{A}(s))$ we infer from Proposition 2.1 and part (i) that

$$
\frac{\partial^{+}}{\partial s} \mathscr{U}(t, s) \phi=\lim _{h \rightarrow 0^{+}} \mathscr{U}(t, s+h) \frac{1}{h}(\phi-\mathscr{U}(s+h, s) \phi)=\mathscr{U}(t, s) \mathbb{A}(s) \phi .
$$

Analogously, (A 25) entails

$$
\frac{\partial^{-}}{\partial s} \mathscr{U}(t, s) \phi=\lim _{h \rightarrow 0^{+}} \mathscr{U}(t, s) \frac{1}{h}(\mathscr{U}(s, s-h) \phi-\phi)=\mathscr{U}(t, s) \mathbb{A}(s) \phi
$$

for $s \in(0, T)$, whence assertion (ii).

Suppose now that the functions $\delta=\delta(z), f=f(t, x), w=w(t, x), P=P(t, x), Q=Q(t, x)$ are suitable enough so that the operator $A$, given by

$$
A(t) u:=-\nabla_{x} \cdot\left(\delta(t, \cdot) \nabla_{x} u\right) \quad \text { with } \quad \delta(t, x):=\delta(f(t, x))
$$

and subject to Neumann boundary conditions, and the function $\mathfrak{b}$, given by

$$
\mathfrak{b}(t, a, x):=\mathfrak{b}(a, w(t, x), P(t, x), Q(t, x)),
$$

satisfy (A 2) and (A 3), respectively, with $\mathfrak{b}$ being Hölder continuous with respect to time. 
Then, according to [29, Prop.2.2], a problem of the form

$$
\left.\begin{array}{rlrl}
\partial_{t} p+\partial_{a} p+A(t) p & =g(t), & & a>0, s<t \leqslant T, \\
p(t, 0) & =2 \int_{0}^{\infty} \mathfrak{b}(t, a) p(t, a) \mathrm{d} a, & & s<t \leqslant T, \\
p(s, a) & =\phi(a), & & a>0
\end{array}\right\}(P)_{s, \phi, g}
$$

with $s \in(0, T), g \in C\left([0, T], \mathbb{L}_{\varrho}\right)$ and $\phi \in \mathbb{L}_{\varrho}$ given, can be rewritten as a Cauchy problem

$$
\left.\begin{array}{rl}
\dot{p}+\mathbb{A}(t) p & =g(t), \quad t \in(s, T], \\
p(s) & =\phi
\end{array}\right\}(C P)_{s, \phi, g}
$$

where the operator $-\mathbb{A}(s)$ is the generator of the semigroup introduced in (A 14). Thus, it is reasonable to define the mild solution $p \in C\left([s, T], \mathbb{L}_{\varrho}\right)$ of $(C P)_{s, \phi, g}$, and hence of $(P)_{s, \phi, g}$, by

$$
p(t):=\mathscr{U}(t, s) \phi+\int_{s}^{t} \mathscr{U}(t, \sigma) g(\sigma) \mathrm{d} \sigma, \quad s \leqslant t \leqslant T .
$$

This definition is justified since classical solutions to $(C P)_{s, \phi, g}$ are necessarily mild solutions. Indeed, if the function $u \in C\left([s, T], \mathbb{L}_{\varrho}\right) \cap C^{1}\left((s, T], \mathbb{L}_{\varrho}\right)$ with $u(t) \in D(\mathbb{A}(t)) \cap \mathbb{W}_{\varrho, \mathscr{B}}^{2}, t \in$ $(s, T]$, is a classical solution of $(C P)_{s, \phi, g}$ with $\phi \in D(\mathbb{A}(s))$, then Proposition A.1 (ii) implies that $u(t)=p(t)$ for $t \in[s, T]$.

\section{Acknowledgment}

This paper was written while I held a position at the Department of Mathematics at Vanderbilt University in Nashville, USA.

\section{References}

[1] Amann, H. (1990) Dynamic theory of quasilinear parabolic equations-II. Reaction-diffusion systems. Diff. Int. Equ. 3, 13-75.

[2] Amann, H. (1991) Multiplication in Sobolev and Besov spaces. In: Nonlinear Analysis: A Tribute in Honour of Giovanni Prodi. Quaderni, Scuola Normale Superiore di Pisa, pp. $27-57$.

[3] Amann, H. (1995) Linear and quasilinear parabolic problems, Vol. I: Abstract Linear Theory. Birkhäuser, Basel, Boston, Berlin.

[4] Amann, H. \& Walker, CH. (2005) Local and global strong solutions to continuous coagulationfragmentation equations with diffusion. J. Diff. Equ. 218, 159-186.

[5] ANDERson, A. R. A. (2005) A hybrid mathematical model of solid tumour invasion: The importance of cell adhesion. Math. Med. Biol. IMA J. 22, 163-186.

[6] Anderson, A. R. A. \& Chaplain, M. A. J. (1998) Continuous and discrete mathematical models of tumour-induced angiogenesis. Bull. Math. Biol. 60, 857-900.

[7] Anderson, A. R. A., Weaver, A. M., Cummings, P. T. \& Quaranta, V. (2006) Tumor morphology and phenotypic evolution driven by selective pressure from the microenvironment. Cell 127, 905-915.

[8] Ayati, B. P., Anderson, A. R. A. \& WebB, G. F. (2006) Computational methods and results for structured multiscale models of tumor invasion. SIAM Multiscale Model. and Simul. 5, $1-20$. 
[9] Bellomo, N. \& Preziosi, L.. (2000) Modelling and mathematical problems related to tumor evolution and its interaction with the immune system. Math. Comput. Model. 32, 413-452.

[10] Carter, S. C. (1965) Principles of cell motility: The direction of cell movement and cancer invasion. Nature 208, 1183-1187.

[11] Carter, S. C. (1967) Haptotaxis and the mechanism of cell motility. Nature 213, 256-260.

[12] Cusulin, C., Iannelli, M. \& Marinoschi, G. (2005) Age-structured diffusion in multi-layer environment. Nonlin. Anal. Real World Appl. 6(6), 207-223.

[13] DiBlasio, G. \& Lamberti, L. (1978) An initial-boundary value problem for age-dependent population diffusion. SIAM J. Appl. Math. 35(3), 593-615.

[14] Dyson, J., Sánchez, E., Villella-Bressan, R., Webb, G. F. (2007) An age and spatially structured model of tumor invasion with haptotaxis. Discr. Cont. Dyn. Sys. B 8(1), 45-60.

[15] Dyson, J., Sánchez, E., Villella-Bressan, R. \& Webb, G. F. (2007) A spatial model of tumor growth with cell age, cell size, and mutation of cell phenotypes. To appear in: Math. Model. Nat. Phenom.

[16] Goldstein, J. A. (1985) Semigroups of Linear Operators and Applications. Oxford Mathematical Monographs, The Clarendon Press, Oxford University Press, New York.

[17] Henry, D. (1981) Geometric theory of semilinear parabolic equations. Lecture Notes in Mathematics 840. Springer, Berlin, Heidelberg, New York.

[18] Kato, T. (1970) Linear evolution equations of "hyperbolic" type. J. Fac. Sc. Univ. Tokyo 25, 241-258.

[19] Kato, T. (1970) Linear evolution equations of "hyperbolic" type II. J. Math. Soc. Japan 25, 648-666.

[20] Kunisch, K., Schappacher, W. \& Webb, G. F. (1985) Nonlinear age-dependent population dynamics with diffusion. Inter. J. Comput. Math. Appl. 11, 155-173.

[21] Langlais, M. (1985) A nonlinear problem in age-dependent population diffusion. SIAM J. Math. Anal. 16(3), 510-529.

[22] Nickel, G. \& RhANDI, A. (1995) On the essential spectral radius of semigroups generated by perturbations of Hille-Yosida operators. Tübinger Berichte zur Funktionalanalysis 4, 207-220.

[23] Orme, M. E. \& Chaplain, M. A. J. (1996) A mathematical model of vascular tumour growth and invasion. Math. Comput. Model. 23, 43-60.

[24] PazY, A. (1983) Semigroups of Linear Operators and Applications to Partial Differential Equations. Springer Verlag, Berlin, New York, Heidelberg.

[25] Quaranta, V., Weaver, A. M., Cummings, P. T. \& Anderson, A. R. A. (2005) Mathematical modeling of cancer: The future of prognosis and treatment. Clinica Chem. 357, 173-179.

[26] Rhandi, A. (1998) Positivity and stability for a population equation with diffusion on $L^{1}$. Positivity 2, 101-113.

[27] Rhandi, A. \& Schnaubelt, R. (1999) Asymptotic behaviour of a non-autonomous population equation with diffusion in $L^{1}$. Disc. Cont. Dyn. Syst. 5, 663-683.

[28] Triebel, H. (1995) Interpolation Theory, Function Spaces, Differential Operators. 2nd ed. Johann Ambrosius Barth, Heidelberg, Leipzig.

[29] WalKer, СH. (2007) Global well-posedness of a haptotaxis model with spatial and age structure. Diff. Int. Eq. 20(9), 1053-1074.

[30] Walker, Ch. \& Webb, G. F. (2007) Global existence of classical solutions for a haptotaxis model. SIAM J. Math. Anal. 38(5), 1694-1713.

[31] Weвb, G. F. (1982) Diffusive age-dependent population models and an application to genetics. Math. Biosci. 61, 1-16.

[32] Webi, G. F. (2008) Population models structured by age, size, and spatial position. In: Magal, P. \& Ruan, S. (editors). Structured Population Models in Biology and Epidemiology. To appear in: Lecture Notes in Mathematics 1936. Berlin. Springer. 